\title{
1 Exploring the miRNA-mediated response to combined stresses 2 in melon plants
}

4 Pascual Villalba-Bermell ${ }^{1}$, Joan Marquez-Molins ${ }^{1}$, María-Carmen Marques ${ }^{1}$, Andrea G.

5 Hernandez-Azurdia ${ }^{1}$, Julia Corell-Sierra ${ }^{1}$, Belén Picó ${ }^{2}$, Antonio J. Monforte ${ }^{3}$, Santiago F.

6 Elena $^{1,4}$, and Gustavo G. Gomez ${ }^{1}$.

7

8

$9{ }^{1}$ Instituto de Biología Integrativa de Sistemas (I²ysBio), Consejo Superior de Investigaciones 10 Científicas (CSIC) - Universitat de València (UV), Parc Científic UV, Catedrático Agustín 11 Escardino 9, Paterna, 46980 Valencia, Spain.

12

$132^{2}$ Instituto de Conservación y Mejora de la Agrodiversidad Valenciana (COMAV), Universitat 14 Politècnica de València (UPV), CPI 8E, Avenida de los Naranjos s/n, 46022 Valencia, Spain.

15

$16{ }^{3}$ Instituto de Biología Molecular y Celular de Plantas (IBMCP), CSIC - UPV, CPI 8E, Avenida de 17 los Naranjos s/n, 46022 Valencia, Spain.

18

$19{ }^{4}$ The Santa Fe Institute, 1399 Hyde Park Road, Santa Fe, NM 87501, USA.

Correspondence

22 Gustavo G. Gomez, Instituto de Biología Integrativa de Sistemas (ISysBio), Consejo Superior de 23 Investigaciones Científicas (CSIC) - Universitat de València (UV), Parc Científic UV, Catedrático 24 Agustín Escardino 9, Paterna, 46980 Valencia, Spain.

25 Email: gustavo.gomez@csic.es

26

27

28 Running title

29 A new viewing in miRNA response to multiple stresses

30

31

32

Keywords

33 Crops production and climate change, miRNAs and stress response in Cucumis melo, RNA 34 regulatory networks, RNA-seq and systems biology. 


\section{Abstract}

2 Climate change has been associated with a higher incidence of combined adverse 3 environmental conditions that can promote a significant decrease in crop productivity.

4 However, knowledge on how a combination of stresses might affect plant development is still

5 scarce. MicroRNAs (miRNAs) have been proposed as potential targets for improving crop-

6 productivity. Here, we have combined deep-sequencing, computational characterization of

7 responsive miRNAs and validation of their regulatory role in a comprehensive analysis of

8 melon's response to several combinations of four stresses (cold, salinity, short day, and

9 infection with a fungus). Twenty-two miRNA families responding to double and/or triple

10 stresses were identified. The regulatory role of the differentially expressed miRNAs was

11 validated by quantitative measurements of the expression of the corresponding target genes. A

12 high proportion (ca. 60\%) of these families (mainly highly conserved miRNAs targeting

13 transcription factors) showed a non-additive response to multiple stresses in comparison with

14 that observed under each one of the stresses individually. Among those miRNAs showing non-

15 additive response to stress-combinations, most interactions were negative suggesting the

16 existence of functional convergence in the miRNA-mediated response to combined stresses.

17 Taken together, our results provide compelling evidences that the response to combined

18 stresses cannot be easily predicted from the study individual stresses. 


\section{INTRODUCTION}

2 During their life cycle, plants are exposed to a wide array of adverse environmental 3 conditions that, in general, limit their normal development and productivity. These 4 complex interactions result in several stress situations that disturb the cell's 5 homeostasis negatively affecting plant-growth. Consequently, stress-induced damages 6 in productivity are the primary cause of extensive agricultural losses worldwide (Priya et 7 al., 2019). Reduction in crop yield due to environmental variations has increased 8 steadily over the last decades. In addition, several production models project a 9 reduction in the yields of major agricultural crops in the future, mostly due to climatic 10 changes (Rosenzweig et al., 2014).

11 Climate change, entailing shifts in temperature, precipitation and atmospheric 12 composition, among other factors, represents a moving target for plant developmental 13 adaptation. In parallel, environmental modifications can favor the development of new

14 plant-pest and/or pathogens or increase the incidence levels of already existing ones.

15 As a consequence of this complex environmental scenario, it is expected that combined 16 abiotic and biotic stresses can affect plants at the level of molecular functions, 17 developmental processes, morphological traits, and physiology, resulting in a significant 18 decrease in crop production and quality (Gray \& Brady, 2016; Morales-Castilla et al., 19 2020).

20 Multiples studies focused on plant responses to individual stresses have been 21 carried out over the last years. However less attention has been paid to the effect that 22 combinations of adverse environmental conditions might exert on plant development. 23 In order to improve crop yield and to meet the growing challenges stemming from rapid 24 population growth, extensive efforts are needed to understand the mechanisms 25 underlying plant responses to simultaneous exposure to multiple stresses (Zhang \& 26 Sonnewald, 2017). Previous works have pointed out that studying stress conditions 27 separately would not allow to infer the expected plant response to multiple stresses. 28 Using Arabidopsis thaliana as experimental model, it was shown that the response to a 29 combination of drought and heat was unique and could not be directly extrapolated 30 from the plant response to each stress applied individually (Rizhsky et al., 2004; Suzuki 31 et al., 2005; Rossel et al., 2007). Similar findings were also reported for a combination 32 of heat and high light intensity in sunflower (Hewezi, Léger \& Gentzbittel, 2008), and 
1 heat and salinity in wheat (Keleş \& Öncel, 2002). Consequently, plant response to

2 combined adverse environmental conditions should be handled as a new state of stress

3 that requires a novel conceptual viewpoint (Mittler \& Blumwald, 2010).

$4 \quad$ In general, plants respond to stress conditions through a complex reprogramming

5 of their transcriptional activities aiming to reduce the impact of stress on their

6 physiological and cell homeostasis. Environmental variations have selected diverse

7 responses among plant lineages, landraces and wild crops relatives. Studies on natural

8 variations can provide novel insights into evolutionary processes modulating stress

9 response (Meyers et al., 2008; Haak et al., 2017). Elucidation of how endogenous

10 regulators and the environment interact during plant development is a long-standing

11 grand challenge in modern biology as well as in crop breeding (Lovell et al., 2015).

12 MicroRNAs (miRNAs) play a versatile role as regulators of gene expression. Plant

13 genes encoding miRNAs are transcribed by RNA polymerase II as primary transcripts

14 harboring a fold back structure that is processed by DICER-LIKE 1 (DCL1) in a duplex (21

15 or 22 nt in length) which once 2'-O-methylated by HEN1 is loaded into an AGO complex

16 (Bartel, 2004; Bologna \& Voinnet, 2014; Reis, Eamens \& Waterhouse, 2015; Achkar,

17 Cambiagno \& Manavella, 2016). miRNAs regulate gene expression by means of

18 sequences complementarity with both RNA and DNA targets (Song, Li, Cao \& Qi, 2019).

19 Their functions include modulation of a vast array of plant biological processes related

20 to grown and development (Bologna \& Voinnet, 2014), including the recovering of the

21 plant-cell homeostasis during exposure to adverse environmental condition (Song et al.,

22 2019; Xu et al., 2019). In addition, it has been recently described that the biogenesis

23 and turnover of certain miRNAs is also susceptible to be controlled by external stimulus

24 (Bustamante et al., 2018; Manavella, Yang \& Palatnik, 2019). Indeed, it has been

25 proposed that miRNAs are ideal targets to be manipulated to improve crop productivity

26 (Tang \& Chu, 2017; Xu et al., 2019). However, most of the described stress-responsive

27 miRNAs come from rice and tomato, as very few miRNAs have been investigated in

28 detail in other crops. Henceforth, additional efforts are needed to decipher the role of

29 miRNA-mediated responses to adverse environmental conditions in other economically

30 relevant crops (Tang \& Chu, 2017).

31 Although, increasing evidences support the role of miRNAs as key modulators of

32 plant response to both biotic (Sun, Niu \& Fan, 2017; Xie et al., 2017; Brant \& Budak, 
12018 ) and abiotic stress conditions (Cervera-Seco et al., 2019; Wang et al., 2020; Cheng

2 et al., 2021; Zhao et al., 2021), research focusing on elucidating the regulatory role of

3 the miRNAs during exposure to combined adverse environmental conditions is still

4 scarce (Xu et al., 2019) and only a few studies considering the effects of an unique

5 combination of stresses have been addressed in soybean (Ning et al., 2019) and A.

6 thaliana (Gupta, Patil, Qamar \& Senthil-Kumar, 2020).

7 Melon (Cucumis melo) is one of the cucurbit crops with more economic impact.

8 Melon has a high adaptability to warm and dry climates, so it can be a target crop to

9 cope with the climate change threats. Previous genetic studies in cucurbits have been

10 focused mainly in fruit quality and disease resistance (Gonzalo \& Monforte 2017).

11 However, the study of the response to combined stress conditions have not been

12 thoroughly addressed in cucurbits. Consequently, there is a lack of consensus protocols,

13 target traits and, therefore, identification of tolerant genotypes to develop efficiently

14 resilient cultivars.

15 Here, we use deep-sequencing, computational approaches and specific miRNA-

16 targets quantification to present a comprehensive functional analysis of miRNA

17 expression profiles in response to one triple (cold, salinity and short day) and five

18 double (cold and drought, cold and salinity, cold and short day, drought and salinity,

19 and drought and infection with the fungus Monosporascus cannonballus) combinations

20 of stress conditions in melon (Cucumis melo), a crop extensively cultivated in semi-arid

21 regions worldwide. The analyzed stress conditions were coincident, in part, with those

22 employed recently to infer the miRNA-mediated regulatory network of response to

23 individual stresses in melon (Sanz-Carbonell et al., 2019; Sanz-Carbonell, Marques,

24 Martinez \& Gomez, 2020). The parallelism between both experimental approaches

25 made possible to unambiguously analyze the effects that the combined adverse

26 environmental conditions have on the accumulation of the stress-responsive miRNAs.

27

282 METHODS

292.1 Plant material, growth conditions, and stress treatments

30 Melon seeds of cv. Piel de Sapo were germinated in Petri dishes at 37 o $\mathrm{C} / 48 \mathrm{~h}$ in

31 darkness followed by 24 h/25 ㅇ (16/8 light/darkness). Melon seedlings were sown in

32 pots and maintained for 10 days under controlled conditions (28 ㅇ C/16 h light and 20 
$1 \stackrel{\circ}{\circ} / 8$ h darkness). At day 11, plants were exposed to six stress-combined treatments

2 (detailed in Table S1). At eleven days post-treatment, the first leaf under the apical end

3 per plant was collected in liquid nitrogen and maintained at -80 ㄷ until processing.

4 Each analyzed sample corresponds to a pool of three treated plants. Three biological

5 replicates were performed per treatment. Leaves recovered from non-treated plants

6 were considered as controls.

7

8 2.2 RNA extraction and small RNA (sRNA) purification and sequencing

9 Total RNA was extracted from leaves ( $\sim .1 \mathrm{~g})$ recovered from treated and control melon

10 as previously described (Sanz-Carbonell et al., 2019; Sanz-Carbonell, Marques, Martinez

11 \& Gomez, 2020). The low-molecular weight RNA (<200 nt) fraction was enriched from

12 total RNA using TOTAL-miRNA (miRNA isolation Kit, REAL) according to the

13 manufacturer's instructions. Production and sequencing of the libraries were carried

14 out by Novogene (https://en.novogene.com). Eighteen cDNA libraries were obtained by

15 following Illumina's recommendations and sequenced in a HiSeq 2000 (Illumina)

16 equipment. Adaptors and low-quality reads were trimmed by using the cutadapt

17 software. For the sake of comparing the results generated in here with those obtained

18 for single stresses, data previously obtained from melon plants exposed to identical

19 single stress conditions for 11 days (Sanz-Carbonell et al., 2019) were also included in

20 the study. Melon miRnA sequences used in this study have been submitted to the

21 genomic repository SRA of the NCBI and are available in the BioProject (PRJNA741881).

22

232.3 RT-qPCR assays

24 To analyze the expression of target genes, total RNA $(1.5 \mu \mathrm{g})$ was subjected to DNase

25 treatment (EN0525, Thermo Scientific ${ }^{\top M}$ ) followed by reverse transcription using

26 RevertAid First Strand CDNA Synthesis Kit (Thermo Scientific ${ }^{\text {TM }}$ ) according to the

27 manufacturer's instructions for use with oligo-dT. cDNAs were amplified by

28 conventional end-point RT-PCR using specific primers to assess for sequence specificity.

29 Then, real-time PCR was performed as described previously (Bustamante et al., 2018).

30 All analyses were done in triplicate on a QuantStudio qPCR instrument (Thermo

31 Scientific ${ }^{\mathrm{TM}}$ ) using a standard protocol. The efficiency of PCR amplification was derived

32 from a standard curve generated by four 10-fold serial dilution points of cDNA obtained 
1 from a mix of all the samples. Relative RNA expression was quantified by the

2 comparative $\Delta \Delta C_{T}$ method (Livak \& Schmittgen, 2001) and normalized to the geometric

3 mean of Profilin (NM_001297545.1) expression. The statistical significance of the

4 observed differences was evaluated by the paired t-test. All primers used were

5 described previously (Sanz-Carbonell et al., 2019).

6

72.4 Bioinformatic analysis of miRNA sequences

8 To study the correlation exhibited by the miRNA expression profiles among the different

9 stresses and their biological replicates, principal component analysis (PCA) was used.

10 PCA was performed using the prcomp function with scaling in the stats $\mathrm{R}$ package $\mathrm{v}$.

11 4.0.4 (R Core Team 2013). Mann-Whitney-Wilcoxon tests were performed to assess for

12 significant differences in the data clusters for Euclidean distances calculated between

13 groups and among groups with the wilcox.test function in the stats R package.

14 Differential expression of melon sRNAs was estimated using three $\mathrm{R}$ packages

15 NOISeq (Tarazona et al., 2015), DESeq2 (Love, Huber \& Anders, 2014) and edgeR

16 (Robinson \& Oshlack, 2010) for pairwise differential expression analysis of expression

17 data. Differentially expressed sRNAs were filtered out using three criteria: (i) $\log _{2}$-fold

18 change $\left|\log _{2} F C\right| \geq 1.25$, (ii) adjusted $p \leq 0.05$ (DESeq2 and edgeR) and probability $\geq 0.95$

19 (NOISeq), and (iii) RPMs $\geq 5$ for at least three libraries in control samples or at least two

20 libraries in any stress. SRNAs identified as responding to stress by the three methods

21 were aligned against miRNA sequences in miRBase (release 22) (Kozomara, Birgaoanu \&

22 Griffiths-Jones, 2019). Only fully homologous miRNAs to previously described mature

23 melon miRNAs and known Viridiplantae miRNAs were kept. Afterwards, these

24 sequences were re-annotated by aligning them against miRNA precursors of melon

25 deposited in miRBase and were considered as known stress-responsive miRNAs.

26 Unaligned sequences were realigned allowing for one mismatch against the melon

27 genome to identify potential precursors. These sequences were also identified as

28 known stress-responsive miRNAs; the rest were discarded. The entire pipeline is shown

29 in Figure $\mathrm{S} 2$.

30 To determine the general sense of the expression for each miRNA family we

31 employed the median value of expression estimated by box-plot analysis of all family-

32 related sequences under each stress condition considering the $\log _{2}$ FC values obtained 
1 by edgeR. The most frequent sequence in each miRNA family and stress were used to

2 generate heatmaps with an $R$ interface to morpheus.js heatmap widget

3 (https://github.com/cmap/morpheus.R).

4

52.5 Analysis of the stress combination effect

6 The expression of reactive miRNAs in response to combined stress conditions can be

7 enfolded in at least one of the three following categories: (i) additive if the observed

8 response to combined stresses is just the sum of the magnitude responses observed for

9 each individual stress, i.e., this represents the null hypothesis of independent actions,

10 (ii) negative if the observed response is smaller than the expected additive response

11 and (iii) positive if the observed value is greater than the expected additive response. In

12 this framework, if a given miRNA shows an additive response upon exposure to two

13 stresses, it can be assumed that both stresses trigger independent miRNA-mediated

14 responses. In contrast, a miRNA showing a significantly negative or positive deviation

15 from the null hypothesis, shall be taken as indicative of a specific response to the

16 combined stresses beyond the simple additive case. To quantitatively test the null

17 hypothesis of additive effects on miRNA-mediated response to stress combinations, we

18 define an stress combination effect (SCE) index that refers to the miRNA response value

19 to combined stresses in comparison to what should be expected from individual stress

20 conditions as $S C E=\left(C+S_{a b}\right)-\left(S_{a}+S_{b}\right)$, where $C$ refers to the means of the

21 normalized reads recovered in control, $S_{a b}$ to the reads observed in plants exposed to

22 combined stresses $a$ and $b$ and $S_{a}$ and $S_{b}$ to the reads arising from each individual stress

23 (Table S6A and Table S6B). For the triple stress condition ( $\left.S_{a b c}\right)$ and additional value $\left(S_{c}\right)$

24 -referred to the means of normalized reads in the additional stress condition $c$ - should

25 be added to the second terms of the equation. Only SCE values with a significant false

26 discovery rate (FDR)-adjusted $p$ value were considered as reliable indicators of effects

27 of stress-combinations onto miRNA accumulation.

28 Reads exhibiting zero means values in any of the analyzed combinations were

29 filtered out. The data associated to the miRNA expression under single stress

30 conditions were extracted from a previous work analyzing the differential expression of

31 melon miRNAs in response to seven biotic and abiotic single stress conditions (Sanz-

32 Carbonell et al. 2019). The statistical significance of these effects was calculated on the 
1 basis of a standard Normal distribution. Then, the 22 stress-responsive miRNA-families

2 were organized in a binary table of presence and absence (Table S7), in which the

3 values one and zero represent, respectively, whether or not a miRNA family has at least

4 a member exhibiting a significant non-additive (positive or negative) effect in response

5 to a combined stress condition. The hclust function in stats R package (v. 4.0.4) was

6 used to compute a hierarchical clustering (HC) specifying Ward linkage (ward.D) as an

7 agglomeration method and using the simple matching coefficient metric to calculate

8 the distance matrix. The statistical significance of the HC was estimated with a Mann-

9 Whitney-Wilcoxon test.

\section{RESULTS}

\subsection{Stress combinations and sRNAs dataset}

13 High-throughput sequencing of sRNAs was performed starting from 22 (three replicates

14 for each stress condition plus four non-treated controls) sRNA libraries constructed with

15 RNA extracted from leaves of melon plants 11 days after exposure to six (five double

16 and one triple) combined stress conditions: (i) cold and drought (C-D), (ii) cold and

17 salinity (C-Sal), (iii) cold and short day (C-SD), (iv) drought and salinity (D-Sal), (v)

18 drought and M. cannonballus infection (D-Mon), and (vi) cold, salinity and short day (C-

19 Sal-SD) (Table S1). Regarding the stress conditions analyzed, we selected abiotic

20 conditions well established as crucial for melon plant development (cold, drought,

21 salinity, and short day) and infection with M. cannonballus, a soil-borne fungal

22 pathogen causing root rot and wilting in melon (Pollack \& Uecker, 1974). Only

23 sequences with size ranging between 20 - 25 nt in length and nonmatching to rRNA,

24 tRNA, snoRNA, and snRNA sequences deposited in the Rfam data base

25 (http://rfam.xfam.org) were further included in this study. A total of 80,620,994 reads

26 (representing 36,836,230 unique sequences) were recovered. The distribution of reads

27 by stress condition is detailed in Table S2.

28 Associations between sRNA expression profiles (considering the different 29 treatments and their biological replicates) were evaluated using PCA. The percentages 30 of variance explained by the first three PCs were $20.4 \%, 17.1 \%$ and $13.8 \%$, respectively 31 (adding up to 51.3\% of the total observed variance). The PCA plot in Figure 1A shows 32 that biological replicates clustered together (attesting for the reproducibility of our 
bioRxiv preprint doi: https://doi.org/10.1101/2021.07.30.454429; this version posted July 31, 2021. The copyright holder for this preprint (which was not certified by peer review) is the author/funder, who has granted bioRxiv a license to display the preprint in perpetuity. It is made available under aCC-BY-NC-ND 4.0 International license.

1 assays) and treatments clearly separated in the PC space with high significance ( $p=$

$\left.25.886 \times 10^{-15}\right)$. The sRNAs exhibited a distribution of read lengths strongly enriched for

324 nt long (45.7\%), followed by similar accumulations of 21 (13.5\%), 22 (12.6\%) and 23

$4 \quad(13.5 \%)$ nt long molecules. As expected, reads of 20 and 25 nt represented the less

5 abundant categories (5.9\% and 8.5\%, respectively) (Figure 1B). These differences in

6 accumulation of different sRNA lengths was statistically significant (2-ways non-

7 parametric ANOVA, Table S3:a $p<10^{-5}$ ). The effect was entirely due to the large

8 enrichment in 24 nt long sRNAs (Dunn's post hoc pairwise tests, Table S3b: $p \leq 0.0134$

9 in all pairwise comparisons) and consistent with what has been previously described in

10 melon (Sattar et al., 2012; Herranz, Navarro, Sommen \& Pallas 2015; Sanz-Carbonell et

11 al., 2019; Sanz-Carbonell, Marques, Martinez \& Gomez, 2020) and other members of

12 the Cucurbitaceae family (Jagadeeswaran et al., 2012). Non-significant differences were

13 found between stress conditions regarding the observed distribution of sRNAs sizes

14 (Table S3a: $p=0.857$ ), nor the interaction between both factors (Table S3a: $p=0.750$ ).

(a)

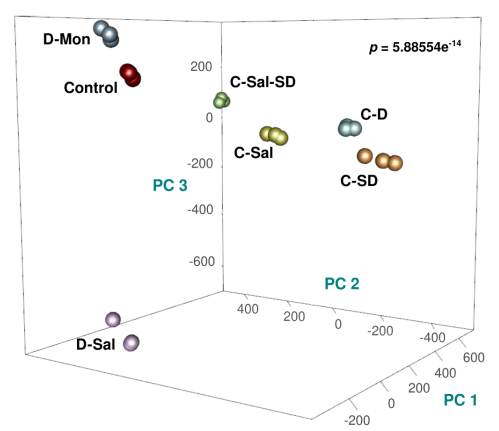

(b)

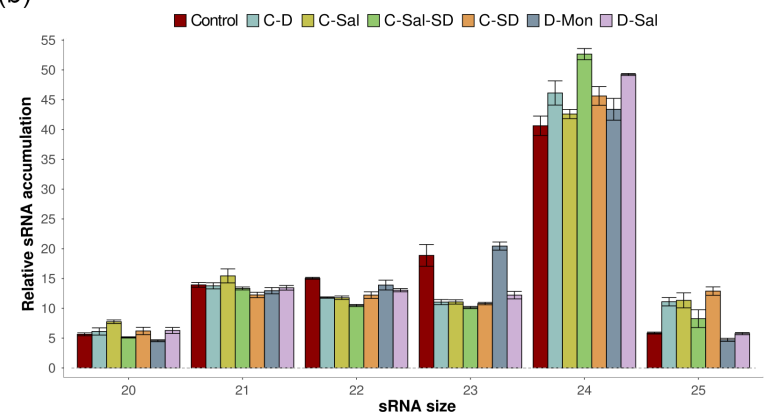

(c)

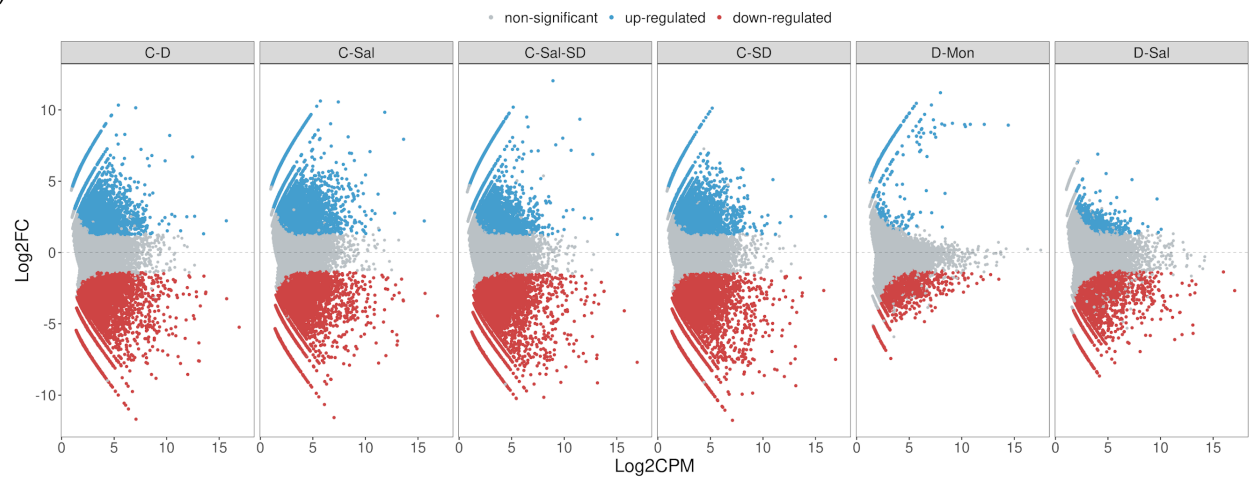

Figure 1 Analysis of the sRNA population. (a) PCA based on sRNAs accumulation in three biological replicates of melon plants exposed to the six stress combined treatments and controls. The statistical significance $(p=5.886 \times 10-14)$ was estimated by Mann-Whitney-Wilcoxon test, considering the inter- and intra-group Euclidean distances. (b) Diagram showing the relative accumulation (and distribution of the total clean reads of melon sRNAs ranging between 20 - $25 \mathrm{nt}$ obtained from the analyzed sequenced libraries. The control and the different analyzed treatments are represented with colors. The shown values represent the sum of all repetitions. Bars indicate the standard error. (c) Graphic representation of the expression values (estimated by edgeR) of sRNA sequences recovered from melon exposed to different stress conditions. The dots indicate the expression value of each sRNA. Red and blue dots indicate 
1 The effect of the stress conditions onto sRNAs accumulation was evaluated by

2 pairwise comparisons between control and treated samples. As described in section 2.4

3 above, only sequences that match the conditions $\left|\log _{2} F C\right| \geq 1.25$ and $p<0.05$, were

4 considered as significantly differentially expressed and retained for subsequent analysis

5 (Figure S1). A total of 35,906 unique reads fulfilled these conditions. The combinations

6 that included cold as one of the stressors showed the most drastic alteration in sRNAs

7 accumulation (21,592 reactive sRNAs in C-D, 20,760 in C-Sal, 23,506 in C-SD and 21,263

8 in C-Sal-SD). In contrast, only 1595 and 3988 differentially expressed sRNAs were

9 identified in plants treated with the combination D-Mon and D-S, respectively (Figure

10 S2B). These results support the notion that exposition to low temperature (in any

11 combination) is the most stressful environmental condition, resulting in the strongest

12 alteration of the sRNA metabolism in melon (Figure 1C).

13

143.2 Combined stresses induce a general decrease of miRNA expression

15 To identify melon miRNAs reactive to combined stress conditions, differentially

16 expressed sRNAs were aligned against miRNA sequences (both mature and precursors)

17 recovered from miRBase (http://www. mirbase.org/). Only sRNAs ranging 20 - 22 nt and

18 fully homologous to database sequences, were considered. Two sequences

19 homologous to mature miR6478 but lacking a known transcript in melon with a

20 canonical hairpin were excluded for subsequent analysis (Figure S1). After filtering, 100

21 unique sequences belonging to 22 known miRNA families were identified as responsive

22 to the combined stress conditions studied (Table S3). In general, all family-related

23 sequences showed a comparable trend of accumulation in response to the stress

24 conditions analyzed (Figure 2A). A sequence-variant of miR398b (down-regulated in C-

25 D treatment, but showing a minority accumulation rate respect to predominant family-

26 related sequences) and the non-canonical miRNAs derived of the alternative processing

27 of miR319 (miR319nc) (Bustamante et al., 2018) and miR159 (miR159nc) (Bologna,

28 Mateos, Bresso \& Palatnik, 2009) precursors (up-regulated in cold-containing

29 combinations and without regulatory activity described yet) showed a discordant

30 response with the family-wise trend. In these two circumstances, the response trend of

31 the more representative family members was considered for ulterior analysis. 
bioRxiv preprint doi: https://doi.org/10.1101/2021.07.30.454429; this version posted July 31, 2021. The copyright holder for this preprint (which was not certified by peer review) is the author/funder, who has granted bioRxiv a license to display the preprint in perpetuity. It is made available under aCC-BY-NC-ND 4.0 International license.

The general response to stress conditions was the down-regulation of miRNAs

2 (Figure 2B). Sequences included in miRNA families miR157, miR159, miR167, miR168,

3 miR319, and miR396 showed significantly decreased accumulation in all the stress

4 conditions analyzed. Diminished accumulation in response to stress was also observed

5 for miR156, miR160 (except under C-Sal-SD), miR164, miR166, miR169 (except for D-

6 Sal), miR171, miR172 (except for D-Sal and D-Mon), miR393 (except for D-Mon),

7 miR394, and miR1515. Finally, miR165 was down-regulated in three stress conditions

8 involving cold (C-SD, C-D and C-Sal). Regarding miRNAs up-regulated in response to

9 stress, the miR398 and miR408 family-related members (except for the reads related to

10 miR398b described above) showed increased accumulation in all stress conditions,

11 whereas miR159 was significantly overexpressed in response to C-SD and C-D and

12 miR397 family was so in plants exposed to C-Sal, C-Sal-SD and D-Mon. Sequences

13 related to miR156, miR166 and miR395 were specifically up-regulated under D-Sal

14 stress.

15

(a)

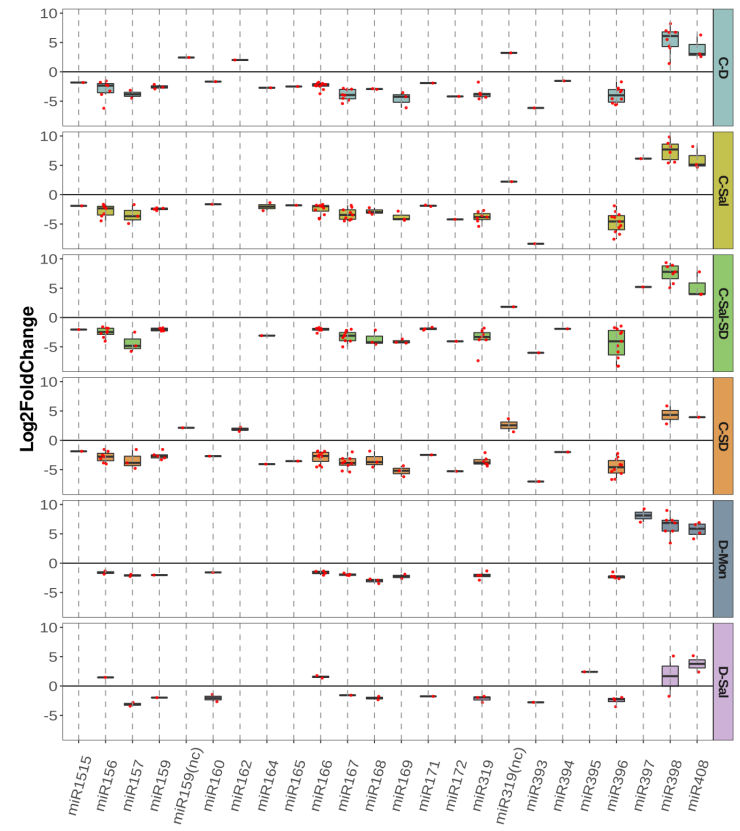

(b)

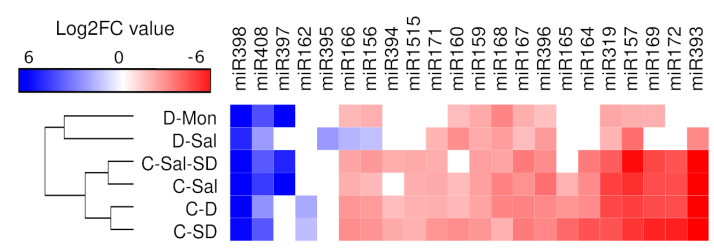

(c)

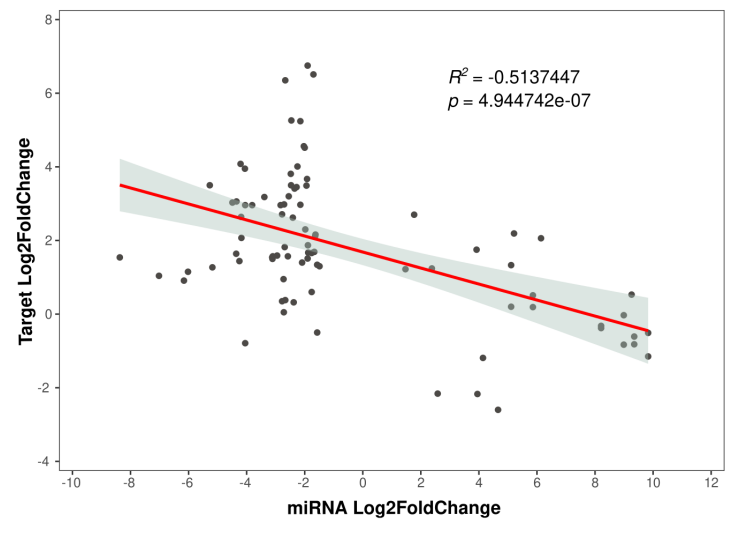

Figure 2 General description of stress-responsive miRNA families: (a) Boxplot analysis showing the general expression value observed for each miRNA-family member. To determine the general sense of the expression for each miRNA family we employed the median value of expression (represented by internal box-line) estimated by boxplot analysis of all family-related sequences. The differential expression values represented in the figure correspond to the log2 FC obtained using edgeR. (b) Heatmap of 22 miRNAs differentially expressed in melon in response to combined stress. The differential expression values represented correspond to the edgeR. (b) Heatmap of 22 miRNAs differentially expressed in melon in response to combined stress. The differential expression values represented correspond to the
median of the log2FC values obtained using edgeR for each miRNA family. (c) Scatter plot showing the significant negative correlation (estimated by Pearson correlation coefficient) between the expression levels of 16 selected stress-responsive miRNAs with differential accumulation determined by sequencing and the accumulation of 
1 The analysis of the miRNA expression focused on each particular stress

2 combination evidenced that cold was the most adverse environmental condition with

3 major impact on miRNA expression in melon. A total of 20 miRNA families were

4 reactive to C-SD and C-D and 19 to C-Sal (Figure 2B and Table S4). While 18 miRNAs

5 families showed differential expression under the combination of three stresses. A

6 weaker response was associated to treatments with D-Sal (14 reactive miRNA families)

7 and D-Mon (13 miRNAs with altered expression). Considering both stress condition and

8 miRNA expression-trend, except miR156 and miR166 (up-regulated in D-Sal and down

9 regulated in the other stress conditions), all miRNAs exhibit a homogenous response to

10 the six combinations of adverse environmental conditions analyzed.

11 It has been recently proposed that certain melon miRNAs are predominantly

12 reactive to diverse biotic and abiotic stress conditions, while other specifically respond

13 to certain stressor and/or expositions time (Sanz-Carbonell, Marques, Martinez \&

14 Gomez, 2020). Based on this particular behavior miRNAs belongings to both different

15 groups were identified as stress responsive miRNAs with broad and narrow response

16 range, respectively, while a third group that exhibit a moderated reactivity in response

17 to stress were identified as intermediates. According to our data, ten miRNA families

18 showed the higher response rate to combined stress, with significant differential

19 expression (either up or down) in the six analyzed conditions (Table S4). Eight of these

20 miRNA families (miR156, miR157, miR166, miR167, miR319, miR396, miR398, and

21 miR408) were mostly coincident with melon miRNAs families classified in the broad

22 response category (generalists), while miR159 and miR168 were previously categorized

23 as intermediates. In contrast, miRNAs with a lower response rate to double and triple

24 stresses (responsive in three o less conditions), pervasively pertained to miRNAs

25 families previously reported as showing specific response to stress conditions in melon.

26 To test the functional role of the miRNAs reactive to combined stresses, we

27 analyzed the correlation between miRNA levels and transcripts accumulation in 16

28 representative miRNA-target modules (Table S5) previously established and validated to

29 occur in melon plants (Bustamante et al., 2018; Sanz-Carbonell et al., 2019; Sanz-

30 Carbonell, Marques, Martinez \& Gomez, 2020). We focused on the miRNAs reactive to

31 at least three different stress conditions (miR156, miR159, miR160, miR164, miR166,

32 miR167, miR169, miR171, miR172, miR319, miR393, miR396, miR397, miR398, and 
1 miR408). As expected, a significant negative correlation ( $r=-0.514,83 \mathrm{df}, p=$

$24.945 \times 10^{-7}$ ) was obtained when the expression values of stress-responsive miRNAs 3 were compared with the accumulation (estimated by RT-qPCR) of their target4 transcripts (Figure 2C).

5

63.3 The miRNA-mediated response to stress combinations cannot be 7 predicted from the response to single stresses

8 To determine the dynamic of the miRNA-mediated response to multiple stress

9 conditions we compare the accumulation levels of stress-responsive miRNAs in plants 10 subjected to the individual stress conditions with those of plants exposed to combined 11 stresses. To do so, we computed SCE as defined in section 2.5 above. Except for the 12 combination C-Sal-SD, the additive effect was predominant in number of unique miRNA 13 sequences in the analyzed stress combinations (65.26\% of the unique reads) (Figure 143 A). However, considering the entire miRNAs population (total reads) a comparable 15 abundance of additive (50.07\%) and non-additive (49.93\%) instances was observed in 16 response to combined stresses. Interestingly, when evaluating only by miRNA family, $1757.58 \%$ had at least a member showing a significant (negative or positive) SCE value 18 (Figure $3 \mathrm{~A}$ and Table S7).

19 Regarding significant non-additive interactions, the stress combination 20 predominantly exerted a negative effect in four (C-Sal, D-Sal, D-Mon, and C-Sal-SD) of 21 the six analyzed treatments (Figure 3B). By contrast, in C-D and C-SD, SCE > 0 values 22 were the most common. Analyzing each stress combination individually, C-SD was the 23 condition in which miRNAs shown the smallest fraction of specific response to 24 combined stresses ( $14.46 \%$ of unique reads, $7.77 \%$ of total reads and $40.91 \%$ of the 25 miRNA families). In contrast, a higher differential interaction (76.47\% for negative and $262.94 \%$ for positive) was observed in response to the triple combinations C-Sal-SD 27 (61.45\% of unique reads, $92.05 \%$ of total reads and $77.27 \%$ of the miRNA families) 28 (Figure 3B). A more general view of the additive and non-additive effects of the 29 combined stresses onto the global population of miRNA-related reads in each analyzed 30 stress condition is showed in the Figure 3C. 
(a)

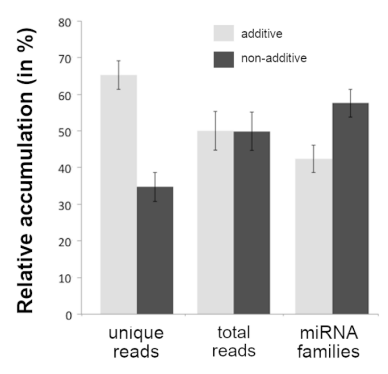

(b)

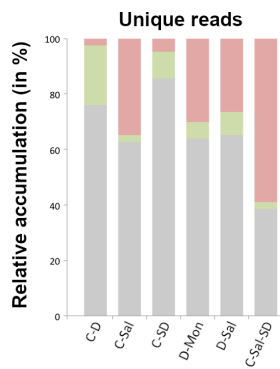

Total reads

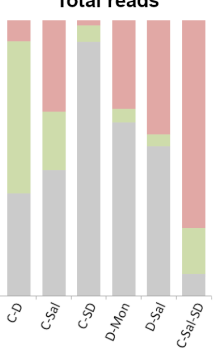

miRNA families

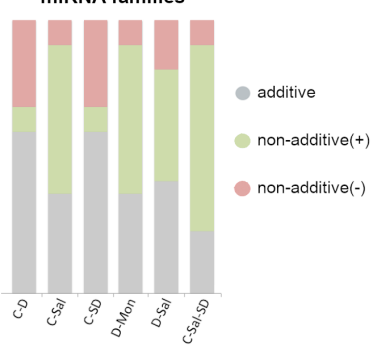

(c) additive $\quad$ non-additive(+) non-additive(-)

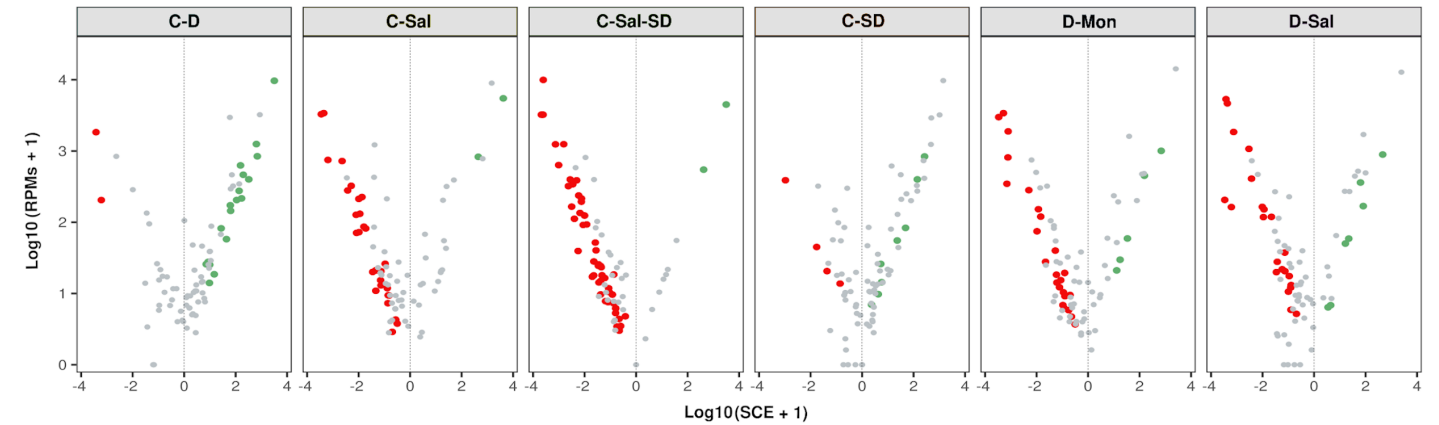

Figure 3 Effects of the stresses combination onto the accumulation rate of stress responsive miRNAs. (a) Graphic representation of the mean percentage for the six analyzed treatments of miRNA related reads that exhibit additive (grey) or non-additive (black) response to combined stress conditions in comparison to single stresses considering unique reads (left columns), total reads (central columns) and miRNA families (right). Bars represent the standard error between means. (b) Detail of the global response rate in each stress condition considering the two (positive or negative) type of possible non-additive response to combined stresses. (c) Volcano plot showing significant positive (green dots) and negative (red dots) SCE values obtained for each miRNA-related read, in response to each combined stress condition. miRNAs with non-significant deviations from the additive null model are in grey. More detailed information is in the Table S6B.

4 Considering the response trend of miRNA family members, we observed that, in

5 general, reads showed a coordinated interaction (SCE positive or negative) in response

6 to the combination of stresses (Figure 4A). Consequently, a negative response was also

7 pervasive under a global miRNA-family viewpoint. Exceptions to this rule were

8 observed for the families miR157 in C-SD and miR159 in D-Sal, that contained members

9 showing both positive and negative SCE values under the indicated stress combination.

10 However, it is worth nothing that the miRNA sequences with a non-coincident trend are

11 minority relative to the other family members (Table S6A). Therefore, in these two

12 specific cases the response trend of the predominant reads was considered as

13 representative of the family behavior for ulterior analysis (Figure 4B). The highest

14 number (17) of miRNA families showing significant SCE values was observed in plants

15 exposed to the triple combinations of stresses, followed by C-Sal and D-Mon (14) and D-

16 Sal (13). In contrast, only nine miRNA-families were identified as significantly 
(a)

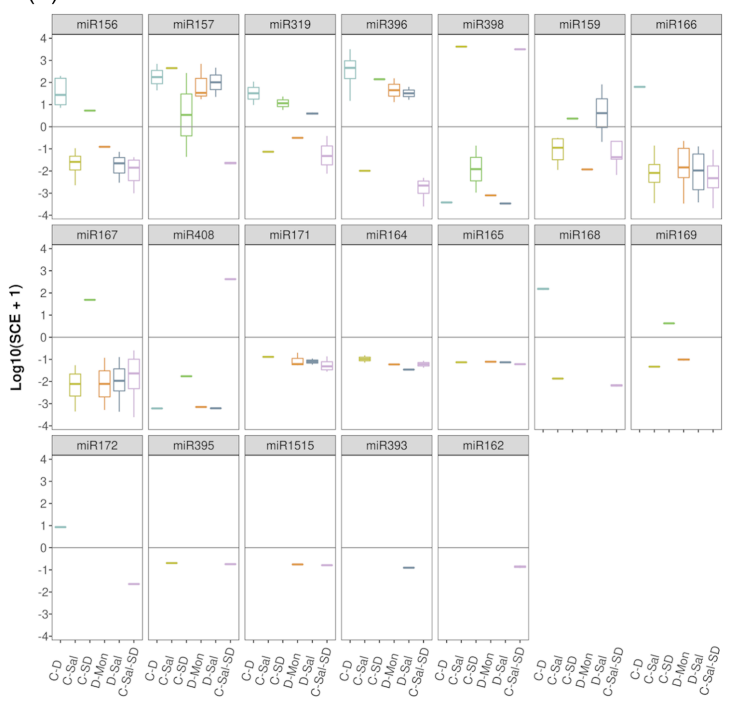

(b)

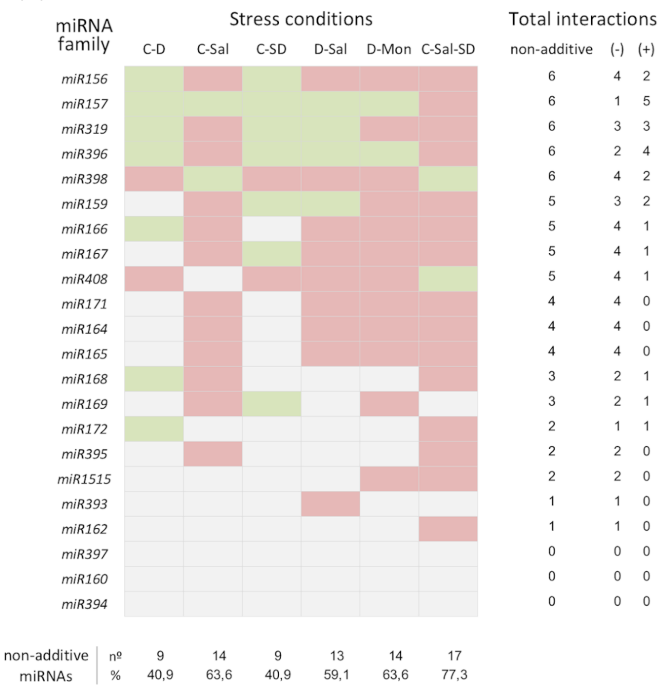

Figure 4 Members in each miRNA family respond in a coordinated manner to combined stresses. (a) Boxplot analysis showing the SCE values for family-miRNA related members in each combined stress condition. To determine the general sense of the effect induced by combined stresses for each miRNA family we employed the median of the SCE values obtained for the totality of the family members (represented by internal box-line). (b) Graphic representation of the global non-additive positive (green) or negative (red) effects associated to combined stresses estimated for each miRNA family in the six stress conditions analyzed here. The number of combined stresses that induce positive and/or negative non-additive responses in each miRNA family is detailed in the right columns. The proportion of miRNA families with non-additive effects in response to each and/or negative non-additive respons
combined stresses is detailed below.

$5 \quad 3.4$ Different miRNAs families act distinctively in response to combined stresses

7 To get further insights into the response of each miRNA family to combined stress 8 conditions, we analyzed the rate of differential response to double and triple stresses.

9 The 22 stress-responsive miRNA-families were organized into a table of presence and 10 absence (Table S8) in which the values one and zero represent, respectively, whether or 11 not a miRNA shows a significant response value (with either positive or negative effect)

12 under a combined stress condition. Members of miR156, miR157, miR319, miR396, and 13 miR398 families showed significant positive or negative SCE in the six stress conditions 14 analyzed here, while miR159, miR166, miR167, and miR408 members accumulate 15 differentially in five stresses combinations. Sequences belonging to miR164, miR165, 16 miR171, and miR393 (with positive or negative SCE in four conditions), miR168 and 17 miR169 (in three), miR172, miR395 and miR1515 (in two) and miR162 (negative effect 18 under C-Sal-SD), showed the lowest differential accumulation in response to the 19 combined stress. Responsive miRNAs included in the miR160, miR394 and miR397 20 families lacked of significant interactions in any the six analyzed stress conditions. 
bioRxiv preprint doi: https://doi.org/10.1101/2021.07.30.454429; this version posted July 31, 2021. The copyright holder for this preprint (which was not certified by peer review) is the author/funder, who has granted bioRxiv a license to display the preprint in perpetuity. It is made available under aCC-BY-NC-ND 4.0 International license.

1 Correlation between miRNA responses (considering miRNA behavior and the

2 different combined treatments) was estimated by multi-cluster analysis (MCA). MCA 3 evidenced that the response values to combined stresses can be organized into three 4 significantly different groups (Figure 5A). The group including miR156, miR157, miR166, 5 miR319, miR396, miR398, and miR408 contained the miRNA families that exclusively 6 show significant non-additive response values (SCE $\neq 0$ values) to combined stress 7 conditions. In contrast, families (miR160, miR162, miR168, miR172, miR394, miR397, 8 miR395, and miR1515) with predominantly independent responses were clustered in 9 the second group. Families of miRNAs in which the proportion of significant (SCE $\neq 0$ 10 values) and non-significant (additive SCE values) response was comparable (miR159, 11 miR164, miR165, miR167, miR169, miR171, and miR393) were also clustered together.

12

(a)

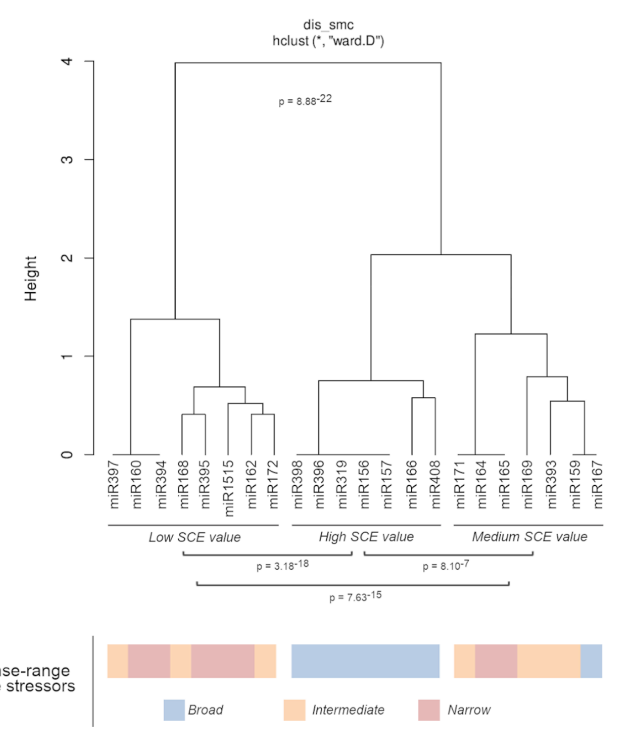

(b)

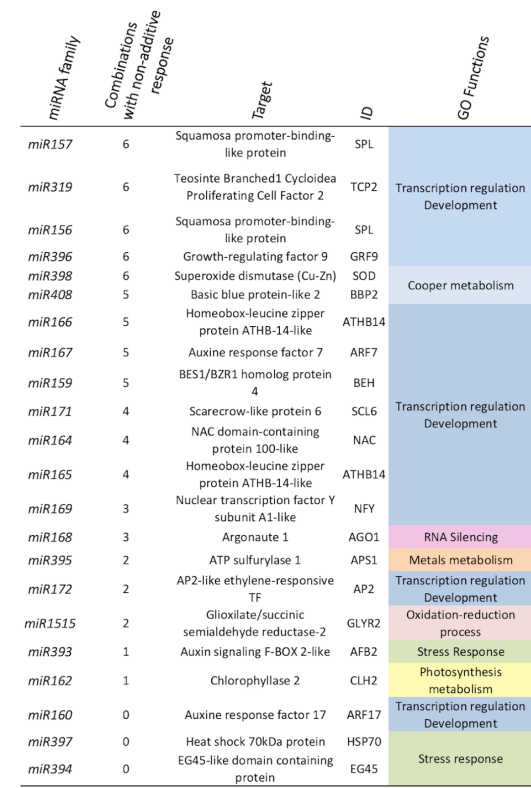

Figure 5 Biological functions of miRNAs with non-additive response to combined stresses. (a) Dendrogram showing the clustering of miRNAs families with at least a member with significant non-additive response to combined stresses in three main groups according to their SCE values in the analyzed stress conditions. The global statistical significance of the identified clusters $\left(p=8.88 \times 10^{-22}\right)$ was estimated by Mann-Whitney-Wilcoxon test considering the inter- and intra-group Euclidean distances. The lower panel shows the response range determined for each miRNA family in response to single stresses with both biotic and abiotic source (using a color scale). (b) Description and detailed information of the targets for miRNAs with

transcripts in $A$. thaliana.

14

Interestingly, all the miRNAs clustered in the group showing significant non-additive expression in response to combined stresses correspond to melon miRNA families

17 already identified as reactive to a broad range of stress (generalists) (Sanz-Carbonell,

18 Marques, Martinez \& Gomez, 2020), while miRNAs characterized by a narrow response 19 range (specialists) are the most frequent class (five out eight) in the group showing 
1 mainly an additive response to double and triple stresses (Figure 5A - lower part).

2 Finally, miRNAs identified previously as intermediates, are mainly (four out seven)

3 included in the group where significant and non-significant response to the combination

4 of stressor was observed at comparable frequencies. The specialist miRNAs exhibit

5 exclusively SCE $<0$ response to double and triple stresses, whereas miRNAs identified as

6 generalists showed an even distribution of significant non-additive responses (20

7 positive and 25 negative SCE values). Intermediate miRNAs, although showed a few

8 miRNAs (five) with positive effects, were predominantly (sixteen miRNA families)

9 characterized by a negative response to the combination of stresses. The relationship

10 between miRNA trend response and stress condition was generally dependent of the

11 specific stress/miRNA interaction, although the miR398 and miR408 families showed a

12 coordinated response in all the analyzed conditions, with the exception of C-Sal.

13 However, a positive response (SCE $=654.96, p=0.04)$ was observed for miR408, in this

14 condition, although was considered as non-significant based in the FDR criterion (Table

15 S6). This specifically coordinated activity of the miR398/miR408 tandem was

16 particularly evident in response to, C-SD and C-Sal-SD in which their response was the

17 opposite to the general trend observed for the remaining miRNA families.

18 Regarding miRNA-regulated targets, it was evident that miRNAs involved in the

19 regulation of transcription factors (TF) associated to plant-development exhibit the

20 higher rate of differential response to combined stress (Figure 4c). In contrast miRNA

21 families expected to modulate the expression of transcripts related (according to GO

22 terms) to a more diverse range of biological functions (RNA silencing, metals

23 metabolism, photosynthesis, response to stress, etc.), showed predominantly a non-

24 significant response to stresses combination.

\section{DISCUSSION}

28 Much effort has been dedicated to elucidating the mechanisms underlying stress

29 response in crops. Although great progress has been made in the last years, including

30 the identification of both protein-coding and non-coding transcripts responsive to

31 different stresses, most studies focused on deciphering the plant regulatory pathways

32 triggered in response to single stress conditions. Alas, no much effort has been devoted 
1 to understand the plant responses to multiple stresses acting simultaneously; a

2 situation that is most common in the wild.

3 Here, we have addressed this question by measuring the miRNA-mediated

4 responses to combined stresses in melon plants exposed to five different double and

5 one triple stressful condition. Our strategy comprises two principal steps, first to

6 identify the miRNA-families responding to double and triple stress conditions. Second,

7 we compared the expression level of such responding miRNAs with the values

8 previously obtained in melon plants exposed to the respective single stresses. This

9 comparative analysis has allowed us to determine how the stress combinations affect

10 the differential expression of miRNAs; disentangling stress-specific responses to general

11 responses. This information enabled the inference of the global structure of the

12 miRNA-mediated differential response to combined stress conditions in melon.

13 The computational analysis identified 22 miRNA families with significant differential

14 expression in response to the analyzed stresses. Regarding their functional role, these

15 reactive families mainly target melon homologous to well-described TFs (e.g.,

16 SPOROCYTELESS, BES1/BZR1 HOMOLOG 4, AUXINE RESPONSE FACTORS (ARF),

17 ARABIDOPSIS THALIANA HOMEOBOX PROTEIN 14, TEOSINTE BRANCHED

18 1/CYCLOIDEA/PROLOFERATING CELL FACTOR, APETALA 2, GENERAL REGULATORY

19 FACTOR (GR), and NUCLER FACTOR Y). This is in agreement with previous observations

20 in other species (A. thaliana, rice, maize, sorghum, sunflower, etc.) in which it has been

21 reported that in general, miRNAs reactive to stress target predominantly TFs (Samad et

22 al., 2017). This reinforces the emerging notion that the role-played by miRNAs during

23 the stress response is evolutionary conserved in plants (Rubio-Somoza \& Weigel, 2011;

24 Megraw, Cumbie, Ivanchenko \& Filichkin, 2016; Sanz-Carbonell, Marques, Martinez \&

25 Gomez, 2020) and emphasizes the potential of miRNAs as targets for improving stress

26 tolerance in crops (Tang \& Chu, 2017; Chaudhary, Grover \& Sharma, 2021). The totality

27 of these stress-responsive miRNA families were coincident with the previously

28 described as reactive in single biotic and abiotic stress conditions in melon (Sanz-

29 Carbonell et al. 2019; Sanz-Carbonell, Marques, Martinez \& Gomez, 2020). The

30 observation that double and triple stresses do not induce the differential accumulation

31 of any miRNA family reactive specifically to combined stress, suggest that (at least

32 under the conditions analyzed here), the miRNA families involved in the response to 
1 stress comprise the general structure that modulate the recovery of the plan-cell

2 homeostasis under both single and combined adverse environmental conditions.

3 Considering the response rate to each stress-combination we observed a more

4 consistent activity in certain miRNA families. Our results evidenced, that melon miRNAs

5 (miR156, miR157, miR166, miR167, miR319, miR396, miR398, and miR408) previously

6 characterized by exhibit differential accumulation in response to a wide range of biotic

7 and abiotic stress conditions in melon, maize and soybean (dubbed as generalists), were

8 differentially expressed in the six analyzed conditions, evidencing a high response

9 range, independently of the stresses combination. Interestingly, miRNAs families

10 reactive to four or less conditions (miR162, miR164, miR165, miR172, miR394, miR397,

11 miR395, and miR1515) predominantly corresponded to miRNAs characterized by

12 exhibiting differential response to specific stresses (specialists). It has been recently

13 suggested that generalists stress-responsive miRNAs might be involved in the

14 modulation of the central steps in the recovery of the cell homeostasis during the

15 exposition to adverse environmental conditions, while specialists families responding to

16 specific stress conditions and/or exposition times had been hypothesized to be involved

17 in the regulation of metabolic processes associated to each particular stressor (Sanz-

18 Carbonell et al. 2019; Sanz-Carbonell, Marques, Martinez \& Gomez, 2020). Assuming

19 this responsive behavior, it is expected that generalist miRNAs were the predominant

20 class reactive to double and triple stresses. Sequences related to generalist miRNA-

21 families are characterized by mainly modulating master regulators or central hubs,

22 predominantly TFs related with plant development (Sanz-Carbonell, Marques, Martinez

23 \& Gomez, 2020). It is well established that alteration in the expression of TF genes

24 normally results in remarkable changes in the global gene expression during plant

25 growth and development (Li et al., 2015). Furthermore, it has been proposed that such

26 TFs might, for example by co-regulatory feedback and feedforward loops miRNA/TF, act

27 as amplifiers of the plant-response to stress (Rubio-Somoza \& Weigel 2011; Megraw et

28 al., 2016; Samad et al., 2017). The generalist class is comprised by miRNAs previously

29 described as reactive to different biotic and/or abiotic stress conditions in diverse plant-

30 species. Several studies support that the module miR156-SPLs besides exhibiting a

31 broad response range to low temperatures in diverse plant-species (Zhou \& Tang,

32 2019), also improves tolerance to salinity, heat and drought in Medicago sativa (Arshad, 
1 et al., 2017; Arshad, Gruber, Wall \& Hannoufa, 2017; Matthews, Arshad \& Hannoufa,

2 2019). Moreover, the interaction between miR396 and GRF is involved in the

3 modulation of the response to diverse biotic (Phytophthora nicotianae) and abiotic

4 (drought, salt, alkali, UV-B radiation, and osmotic unbalance) stress conditions (Gao et

5 al., 2010; Kim et al., 2012; Casadevall et al., 2013; Chen, Luan \& Zhai, 2015). Cotton

6 plants overexpressing miR157 suppressed the auxin signal and showed enhanced

7 sensitivity to heat (Ding et al., 2017). Recent studies evidenced a critical function for

8 miR166 in tolerance to abiotic stresses in maize (Li et al., 2020) and cadmium-induced

9 toxicity in rice (Ding et al., 2018). By means of transgenic approaches it was established

10 that miR167 acts as transcriptional regulator in response to bacterial infection (Jodder,

11 Basak, Das \& Kundu, 2017) and temperature-induced stress in tomato plants (Jodder et

12 al., 2018). Multiple evidences obtained by both sRNA-sequencing and transgenic

13 approaches, support the role of members of the miR319-family, an ancient miRNA

14 conserved across plant species ranging from mosses to higher plants, as a key

15 modulator of the plant-environment interrelation (at biotic and abiotic level) in

16 monocotyledonous and dicotyledonous species (Bustamante et al., 2018; Liu et al.,

17 2019; Shi et al., 2019; Wu, Qi, Meng \& Jin, 2020; Fang et al., 2021; Joshi, Chauhan \&

18 Das, 2021). Finally, regarding miR398 and miR408 families, it was recently proposed

19 that these conserved miRNAs, involved in the maintenance of the cooper homeostasis

20 in plants, might be also involved in the systemic signaling of the response to biotic and

21 abiotic stresses (Burkhead et al., 2009; Sanz-Carbonell, Marques, Martinez \& Gomez,

22 2020).

23 Upon determining the melon miRNAs responsive to combined stress conditions, we

24 attempted to analyze whether the expression of these stress-responsive miRNAs was

25 different in comparison with that observed under each one of the stresses individually.

26 Our conceptual premise assumes that miRNAs that did not show a significant

27 differential (positive or negative) response to combined stresses exhibit an independent

28 behavior to the combination of the stress conditions. The obtained results

29 demonstrated that in a considerable proportion of the analyzed miRNA-stress

30 combinations (59.85\%), the stress-responsive miRNAs families exhibit a differential

31 response to the action of combined stresses. This evidences that, although the miRNAs

32 involved in the regulation of the response to a particular stress combination are 
1 coincident with such described under individual stresses, the regulatory effects exerted

2 on their targets is considerably different when the plant is exposed to a combination of

3 adverse environmental conditions.

$4 \quad$ Considering in detail the differentially reactive miRNAs, we observed that generalist

5 miRNAs showed the higher rate of differential accumulation (compared to the observed

6 respect the response to single stresses) in response to combined adverse

7 environmental conditions. Thus, supporting that the biosynthesis and/or processing of

8 such miRNA-families is particularly (and differentially) susceptible to the combined

9 exposition to two or three stress conditions. In contrast, the data obtained when

10 miRNAs identified previously as specialists were analyzed evidenced that the expression

11 of this class de miRNA families is predominantly independent of the effects of the

12 combined-stresses and corresponds principally to the expression levels observed in

13 response to each stressor individually. This functional behavior of responsive miRNAs

14 to combined stresses is compatible with the architecture of the miRNA-mediated

15 regulatory network of response to adverse environmental stimuli described recently in

16 melon (Sanz-Carbonell et al. 2019; Sanz-Carbonell, Marques, Martinez \& Gomez, 2020).

17 Structurally, this network is characterized by exhibiting a central core of highly

18 connected miRNAs (generalist), and another peripheral layer comprised of miRNA

19 families with lower connectivity (specialists) (Figure 6A). According to this structure, it

20 is expected that the expression of generalist miRNAs (highly interconnected and

21 reactive to a broad range of stress conditions) might be differentially affected (either

22 positively or negatively) by the incidence of two or more distinct stresses (Figure 6B). In

23 contrast, specialist miRNA-families (with low connectivity and reactive to particular

24 stress conditions) remain functionally independent to the effects of additional non-

25 related stresses, and respond mainly to the exposition to combined stress conditions in

26 additive (non-differential) manner (Figure 6A). The observation that the architecture of

27 the miRNA-mediated regulatory network of response to stress in melon is able to

28 predict the predominant reactivity rate of the miRNA-response to combined stresses,

29 provide additional robustness to this inferred regulatory structure involved in the

30 miRNA-mediated modulation of plant-environment interactions. Furthermore, the fact

31 that a structurally comparable miRNA-networks of response to stress has been also

32 proposed in rice and soybean plants exposed to diverse biotic and abiotic stress 
bioRxiv preprint doi: https://doi.org/10.1101/2021.07.30.454429; this version posted July 31, 2021. The copyright holder for this preprint (which was not certified by peer review) is the author/funder, who has granted bioRxiv a license to display the preprint in perpetuity. It is made available under aCC-BY-NC-ND 4.0 International license.

1 conditions (Sanz-Carbonell, Marques, Martinez \& Gomez, 2020), allows to speculate 2 about the possibility that the response pattern to combined stresses observed in melon 3 may well be extended to another crops.

(a)

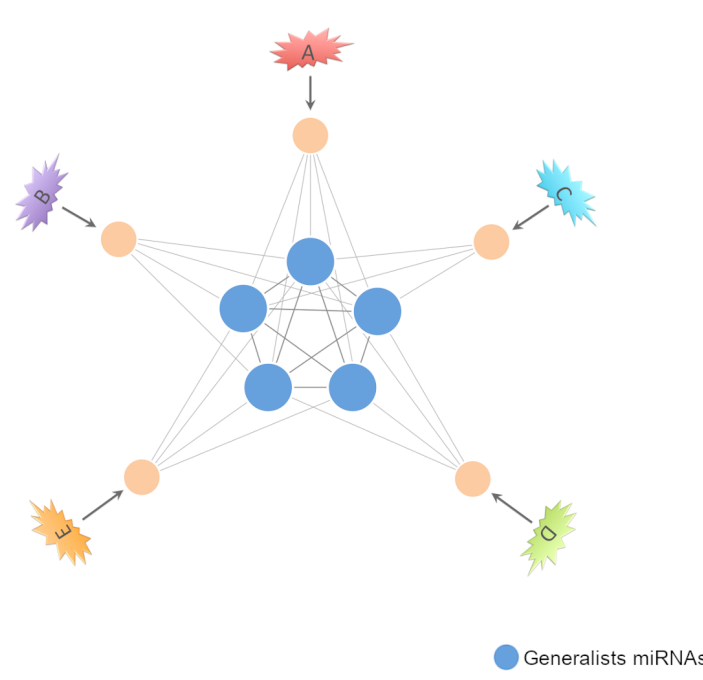

(b) Exposition to combined stressors

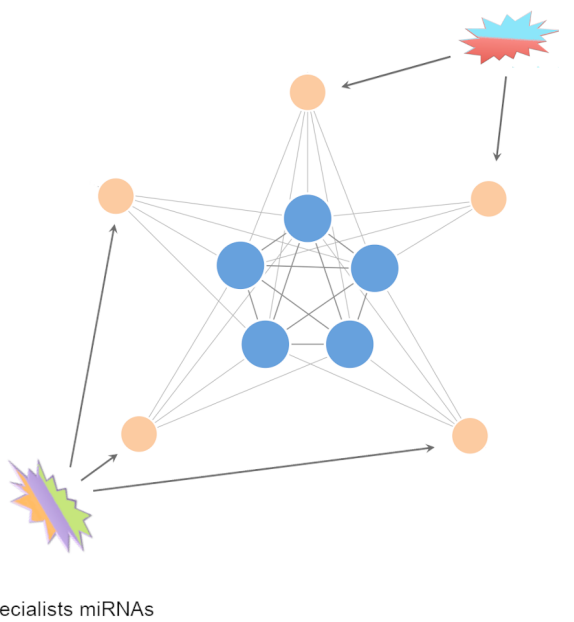

Figure 6: Proposed model to explain predominant non-additive response in certain miRNAs families. (a) Simplified graphic representation of the proposed miRNA-mediated network of response to stress in melon (Sanz-Carbonell et al., 2019; Sanz-Carbonell, Marqués, Martínez \& Gómez, 2020). Blue nodes represent highly connected miRNAs with a broad response range to biotic and/or abiotic stress conditions (generalists). Orange nodes represent miRNAs reactive to specific stress conditions (specialists). (b) When the network is exposed to double or triple stress conditions is expected that the stresses combinations should not affect specialist miRNAs (poorly connected between them) and consequently they exhibit additive SCE values (comparable to the resultant of the sum of both individual responses). In contrast, generalist miRNAs (highly interconnected) respond to stresses combination in a differential (non-additive) manner, related to each stress combination.

In general, the transcripts of well-established TFs were the targets modulated by miRNAs with significant non-additive effects in response to combined stresses, 8 reinforcing the key role assumed for the circuits miRNA-TF in the regulation of the 9 stress response in plants (Rubio-Somoza \& Weigel, 2011). Regarding the trend of the 10 global differential miRNA-mediated response to combined stresses negative values 11 were the most abundant. Response values lower than the expected for stress12 independent effects might be initially assumed as an indicative of functional 13 convergence in the miRNA-mediated response to combined stresses. It has been 14 recently suggested that specific developmental events may be usually modulated by 15 diverse miRNAs in rice (Tang \& Chu, 2017). In this proposed model, miRNAs functionally 16 converged via direct or indirect interaction between their targets. It is well established 17 that osa-miR393 regulate the auxin receptors OsTIR1 and OSAFB2, both involved in the 18 ubiquitin-mediated degradation of specific substrates during auxin signaling (Bian et al., 
1 2012; Li et al., 2016). Furthermore, osa-miR160 and osa-miR167 modulate the

2 expression of at least three ARF transcripts (OSARF8, OsARF16 and OsARF18) (Yang,

3 Han, Yoon \& Lee, 2006; Li et al., 2014; Huang, Li \& Zhao, 2016). Interestingly, cmel-

4 miR393 and cmel-miR167 exhibit a predominant negative differential response

5 (assumed as indicator of functional convergence) to the combined stresses analyzed

6 here. Further studies are needed to determine the existence of a potential functional

7 convergence in the miRNA-mediated response to multiple stresses.

8 Altogether, our results provide additional support to the anticipated notion that

9 plants may use the miRNA-mediated regulation as pivotal mechanism to recover the

10 cell homeostasis in response to both simple and combined stresses (Zhang, 2015;

11 Samad et al., 2017; Zhu et al., 2019; Zhou et al., 2020). The confirmation that the

12 previously described as generalist miRNAs are also the predominant components of the

13 global miRNA-mediated response to combined stress conditions highlights the

14 possibility that this class de miRNAs may emerge as a valuable breeding-target for

15 improving, in the near future, crop tolerance to the multiple adverse environmental

16 conditions associated to climate change.

17

\section{ACKNOWLEDGEMENTS}

19 J.M.M. is recipient of a predoctoral contract ACIF-2017-114 from the Generalitat 20 Valenciana.

21

22 CONFLICT OF INTEREST

23 The authors do not have any conflict of interest to declare.

24

\section{AUTHOR CONTRIBUTIONS}

26 PVB: Performed and designed computational analysis, prepared figures, and discussed

27 the results. JMM: Analyzed the results, prepared figures and contributed to write the

28 manuscript. MCM: Conceived and performed RT-qPCR analyses and discussed the

29 results. AGHA: performed RT-qPCR analysis. JCS: Performed computational analysis.

30 BP: Provide the Monosporascus isolate and contributed to design the stress treatments.

31 AJM: Provided melon seeds and contributed to design the stress treatments. SFE:

32 Conceived and perform the estimation of the SCE values and revised the manuscript.

33 GGG: Conceived and designed the experiments, analyze the results and drafted the

34 manuscript. Manuscript review: All authors read and approved the final manuscript. 
1

\section{REFERENCE}

Achkar N.P., Cambiagno D.A. \& Manavella P.A. (2016) miRNA Biogenesis: A Dynamic Pathway. Trends in Plant Science.

Arshad M., Feyissa B.A., Amyot L., Aung B. \& Hannoufa A. (2017a) MicroRNA156 improves drought stress tolerance in alfalfa (Medicago sativa) by silencing SPL13. Plant Science 258, 122-136.

Arshad M., Gruber M.Y., Wall K. \& Hannoufa A. (2017b) An Insight into microRNA156 Role in Salinity Stress Responses of Alfalfa . Frontiers in Plant Science 8, 356.

Bartel D.P. (2004) MicroRNAs: Genomics, Biogenesis, Mechanism, and Function. Cell $116,281-297$.

Bian H., Xie Y., Guo F., Han N., Ma S., Zeng Z., ... Zhu M. (2012) Distinctive expression patterns and roles of the miRNA393/TIR1 homolog module in regulating flag leaf inclination and primary and crown root growth in rice (Oryza sativa). New Phytologist 196, 149-161.

Bologna N.G., Mateos J.L., Bresso E.G. \& Palatnik J.F. (2009) A loop-to-base processing mechanism underlies the biogenesis of plant microRNAs miR319 and miR159. The EMBO Journal 28, 3646-3656.

Bologna N.G. \& Voinnet O. (2014) The Diversity, Biogenesis, and Activities of Endogenous Silencing Small RNAs in Arabidopsis . Annual Review of Plant Biology 65, 473-503.

Brant E.J. \& Budak H. (2018) Plant small non-coding RNAs and their roles in biotic stresses. Frontiers in Plant Science 9, 1038.

Burkhead J.L., Gogolin Reynolds K.A., Abdel-Ghany S.E., Cohu C.M. \& Pilon M. (2009) Copper homeostasis. New Phytologist 182, 799-816.

Bustamante A., Marques M.C., Sanz-Carbonell A., Mulet J.M. \& Gomez G. (2018) Alternative processing of its precursor is related to miR319 decreasing in melon plants exposed to cold. Scientific Reports 8.

Casadevall R., Rodriguez R.E., Debernardi J.M., Palatnik J.F. \& Casati P. (2013) Repression of Growth Regulating Factors by the MicroRNA396 Inhibits Cell Proliferation by UV-B Radiation in \&lt;em\&gt;Arabidopsis\&It;/em\&gt; Leaves. The Plant Cel/ 25, 3570 LP -3583.

Cervera-Seco L., Marques M.A.C., Sanz-Carbonell A., Marquez-Molins J., Carbonell A., 
Darós J.A. \& Gomez G. (2019) Identification and Characterization of StressResponsive TAS3-Derived TasiRNAs in Melon. Plant \& Cell Physiology 60.

Chaudhary S., Grover A. \& Sharma P.C. (2021) MicroRNAs: Potential Targets for Developing Stress-Tolerant Crops. Life 11.

Chen L., Luan Y. \& Zhai J. (2015) Sp-miR396a-5p acts as a stress-responsive genes regulator by conferring tolerance to abiotic stresses and susceptibility to Phytophthora nicotianae infection in transgenic tobacco. Plant Cell Reports 34, 2013-2025.

Cheng X., He Q., Tang S., Wang H., Zhang X., Lv M., ... Liu J. (2021) The miR172/IDS1 signaling module confers salt tolerance through maintaining ROS homeostasis in cereal crops. New Phytologist $\mathrm{n} / \mathrm{a}$.

Ding Y., Gong S., Wang Y., Wang F., Bao H., Sun J., ... Zhu C. (2018) MicroRNA166 Modulates Cadmium Tolerance and Accumulation in Rice. Plant Physiology 177, 1691-1703.

Ding Y., Ma Y., Liu N., Xu J., Hu Q., Li Y., ... Zhang X. (2017) microRNAs involved in auxin signalling modulate male sterility under high-temperature stress in cotton (Gossypium hirsutum). The Plant Journa/ 91, 977-994.

Fang Y., Zheng Y., Lu W., Li J., Duan Y., Zhang S. \& Wang Y. (2021) Roles of miR319regulated TCPs in plant development and response to abiotic stress. The Crop Journa/9, 17-28.

Gao P., Bai X., Yang L., Lv D., Li Y., Cai H., ... Zhu Y. (2010) Over-expression of osaMIR396c decreases salt and alkali stress tolerance. Planta 231, 991-1001.

Gonzalo M. \& Monforte A. (2017) Genetic Mapping of Complex Traits. Genomics: Essential Methods, 269-290.

Gray S.B. \& Brady S.M. (2016) Plant developmental responses to climate change. Developmental Biology 419, 64-77.

Gupta A., Patil M., Qamar A. \& Senthil-Kumar M. (2020) ath-miR164c influences plant responses to the combined stress of drought and bacterial infection by regulating proline metabolism. Environmental and Experimental Botany 172, 103998. Haak D.C., Fukao T., Grene R., Hua Z., Ivanov R., Perrella G. \& Li S. (2017) Multilevel Regulation of Abiotic Stress Responses in Plants. Frontiers in Plant Science 8, 1564. 
1 Herranz M.C., Navarro J.A., Sommen E. \& Pallas V. (2015) Comparative analysis among

2 the small RNA populations of source, sink and conductive tissues in two different plant-virus pathosystems. BMC Genomics 16, 117.

Hewezi T., Léger M. \& Gentzbittel L. (2008) A Comprehensive Analysis of the Combined Effects of High Light and High Temperature Stresses on Gene Expression in Sunflower. Annals of Botany 102, 127-140.

Huang J., Li Z. \& Zhao D. (2016) Deregulation of the OsmiR160 Target Gene OsARF18 Causes Growth and Developmental Defects with an Alteration of Auxin Signaling in Rice. Scientific Reports 6, 29938.

Jagadeeswaran G., Nimmakayala P., Zheng Y., Gowdu K., Reddy U.K. \& Sunkar R. (2012) Characterization of the small RNA component of leaves and fruits from four different cucurbit species. BMC Genomics 13, 329.

Jodder J., Basak S., Das R. \& Kundu P. (2017) Coherent regulation of miR167a biogenesis and expression of auxin signaling pathway genes during bacterial stress in tomato. Physiological and Molecular Plant Pathology 100, 97-105.

Jodder J., Das R., Sarkar D., Bhattacharjee P. \& Kundu P. (2018) Distinct transcriptional and processing regulations control miR167a level in tomato during stress. RNA Biology 15, 130-143.

Joshi G.A. nee, Chauhan C. \& Das S. (2021) Sequence and functional analysis of MIR319 promoter homologs from Brassica juncea reveals regulatory diversification and altered expression under stress. Molecular Genetics and Genomics.

Keleş Y. \& Öncel I. (2002) Response of antioxidative defence system to temperature and water stress combinations in wheat seedlings. Plant Science 163, 783-790.

Kim J.-S., Mizoi J., Kidokoro S., Maruyama K., Nakajima J., Nakashima K., ... YamaguchiShinozaki K. (2012) \&lt;em\&gt;Arabidopsis\&It;/em\&gt; GROWTH-REGULATING FACTOR7 Functions as a Transcriptional Repressor of Abscisic Acid- and Osmotic Stress-Responsive Genes, Including \&lt;em\&gt;DREB2A\&lt;/em\&gt; The Plant Cell 24, 3393 LP - 3405.

Kozomara A., Birgaoanu M. \& Griffiths-Jones S. (2019) miRBase: from microRNA sequences to function. Nucleic Acids Research 47, D155-D162.

Li J., Han S., Ding X., He T., Dai J., Yang S. \& Gai J. (2015) Comparative Transcriptome Analysis between the Cytoplasmic Male Sterile Line NJCMS1A and Its Maintainer 
NJCMS1B in Soybean (Glycine max (L.) Merr.). PLOS ONE 10, e0126771.

Li N., Yang T., Guo Z., Wang Q., Chai M., Wu M., ... Zhang Z. (2020) Maize microRNA166 Inactivation Confers Plant Development and Abiotic Stress Resistance. International journal of molecular sciences 21, 9506.

Li X., Xia K., Liang Z., Chen K., Gao C. \& Zhang M. (2016) MicroRNA393 is involved in nitrogen-promoted rice tillering through regulation of auxin signal transduction in axillary buds. Scientific Reports 6, 32158.

Li Y., Lu Y.-G., Shi Y., Wu L., Xu Y.-J., Huang F., ... Wang W.-M. (2014) Multiple Rice MicroRNAs Are Involved in Immunity against the Blast Fungus Magnaporthe oryzae . Plant Physiology 164, 1077-1092.

Liu Y., Li D., Yan J., Wang K., Luo H. \& Zhang W. (2019) MiR319 mediated salt tolerance by ethylene. Plant biotechnology journa/ 17, 2370-2383.

Livak K.J. \& Schmittgen T.D. (2001) Analysis of Relative Gene Expression Data Using Real-Time Quantitative PCR and the 2- $\triangle \Delta C T$ Method. Methods 25, 402-408.

Love M.I., Huber W. \& Anders S. (2014) Moderated estimation of fold change and dispersion for RNA-seq data with DESeq2. Genome Biology 15, 550.

Lovell J.T., Mullen J.L., Lowry D.B., Awole K., Richards J.H., Sen S., ... McKay J.K. (2015) Exploiting Differential Gene Expression and Epistasis to Discover Candidate Genes for Drought-Associated QTLs in \&lt;em\&gt;Arabidopsis thaliana\&lt;/em\&gt; The Plant Cel/ 27, 969 LP - 983.

Manavella P.A., Yang S.W. \& Palatnik J. (2019) Keep calm and carry on: miRNA biogenesis under stress. The Plant Journa/99, 832-843.

Matthews C., Arshad M. \& Hannoufa A. (2019) Alfalfa response to heat stress is

$$
\text { modulated by microRNA156. Physiologia Plantarum 165, 830-842. }
$$

Megraw M., Cumbie J.S., Ivanchenko M.G. \& Filichkin S.A. (2016) Small Genetic Circuits and MicroRNAs: Big Players in Polymerase II Transcriptional Control in Plants. The Plant Cel/ 28, 286 LP - 303.

Meyers B.C., Axtell M.J., Bartel B., Bartel D.P., Baulcombe D., Bowman J.L., ... Zhu J.-K. 3190.

Mittler R. \& Blumwald E. (2010) Genetic Engineering for Modern Agriculture: Challenges and Perspectives. Annual Review of Plant Biology 61, 443-462. 
1 Morales-Castilla I., García de Cortázar-Atauri I., Cook B.I., Lacombe T., Parker A., van

2

3

4

5

6

7

8

9

10

11 Leeuwen C., ... Wolkovich E.M. (2020) Diversity buffers winegrowing regions from climate change losses. Proceedings of the National Academy of Sciences of the United States of America 117, 2864-2869.

5 Ning L.-H., Du W., Song H.-N., Shao H.-B., Qi W.-C., Sheteiwy M.S.A. \& Yu D. (2019) Identification of responsive miRNAs involved in combination stresses of phosphate starvation and salt stress in soybean root. Environmental and Experimental Botany $167,103823$.

Pollack F.G. \& Uecker F.A. (1974) Monosporascus cannonballus an Unusual Ascomycete in Cantaloupe Roots. Mycologia 66, 346-349.

Priya M., Dhanker O.P., Siddique K.H.M., HanumanthaRao B., Nair R.M., Pandey S., ... Nayyar H. (2019) Drought and heat stress-related proteins: an update about their functional relevance in imparting stress tolerance in agricultural crops. Theoretical and Applied Genetics 132, 1607-1638.

Reis R.S., Eamens A.L. \& Waterhouse P.M. (2015) Missing Pieces in the Puzzle of Plant MicroRNAs. Trends in Plant Science 20, 721-728.

Rizhsky L., Liang H., Shuman J., Shulaev V., Davletova S. \& Mittler R. (2004) When Defense Pathways Collide. The Response of Arabidopsis to a Combination of Drought and Heat Stress. Plant Physiology 134, 1683-1696.

Robinson M.D. \& Oshlack A. (2010) A scaling normalization method for differential expression analysis of RNA-seq data. Genome Biology 11, R25.

Rosenzweig C., Elliott J., Deryng D., Ruane A.C., Müller C., Arneth A., ... Jones J.W. (2014) Assessing agricultural risks of climate change in the 21st century in a global gridded crop model intercomparison. Proceedings of the National Academy of Sciences 111,3268 LP -3273.

Rossel J.B., Wilson P.B., Hussain D., Woo N.S., Gordon M.J., Mewett O.P., ... Pogson B.J. (2007) Systemic and Intracellular Responses to Photooxidative Stress in Arabidopsis. The Plant Cell 19, 4091-4110.

Rubio-Somoza I. \& Weigel D. (2011) MicroRNA networks and developmental plasticity in plants. Trends in Plant Science 16, 258-264.

Samad A.F.A., Sajad M., Nazaruddin N., Fauzi I.A., Murad A.M.A., Zainal Z. \& Ismail I. (2017) MicroRNA and Transcription Factor: Key Players in Plant Regulatory 
Network. Frontiers in plant science 8, 565.

Sanz-Carbonell A., Marques M.C., Bustamante A., Fares M.A., Rodrigo G. \& Gomez G. (2019a) Inferring the regulatory network of the miRNA-mediated response to biotic and abiotic stress in melon. BMC Plant Biology 19.

Sanz-Carbonell A., Marques M.C., Martinez G. \& Gomez G. (2019b) Dynamic architecture and regulatory implications of the miRNA network underlying the response to stress in melon. RNA Biology, 1-17.

Sattar S., Song Y., Anstead J.A., Sunkar R. \& Thompson G.A. (2012) Cucumis melo MicroRNA Expression Profile During Aphid Herbivory in a Resistant and Susceptible Interaction. Molecular Plant-Microbe Interactions ${ }^{\circledR} 25,839-848$.

Shi X.P., Jiang F.L., Wen J.Q., Cui S.Y., Zhou Y.Z. \& Wu Z. (2019) MicroRNA319 family members play an important role in Solanum habrochaites and S. Iycopersicum responses to chilling and heat stresses. Biologia plantarum 63, 200-209.

Song X., Li Y., Cao X. \& Qi Y. (2019) MicroRNAs and Their Regulatory Roles in PlantEnvironment Interactions. Annual Review of Plant Biology 70, 489-525.

Sun Y., Niu X. \& Fan M. (2017) Genome-wide identification of cucumber green mottle mosaic virus-responsive microRNAs in watermelon. Archives of Virology 162, 2591-2602.

Suzuki N., Rizhsky L., Liang H., Shuman J., Shulaev V. \& Mittler R. (2005) Enhanced Tolerance to Environmental Stress in Transgenic Plants Expressing the Transcriptional Coactivator Multiprotein Bridging Factor 1c. Plant Physiology 139, $1313-1322$.

Tang J. \& Chu C. (2017) MicroRNAs in crop improvement: fine-tuners for complex traits. Nature Plants 3, 17077.

Tarazona S., Furió-Tarí P., Turrà D., Pietro A. Di, Nueda M.J., Ferrer A. \& Conesa A. (2015) Data quality aware analysis of differential expression in RNA-seq with NOISeq R/Bioc package. Nucleic Acids Research 43, e140-e140.

Wang Y., Feng C., Zhai Z., Peng X., Wang Y., Sun Y., ... Li T. (2020) The Apple microR171iSCARECROW-LIKE PROTEINS26.1 Module Enhances Drought Stress Tolerance by Integrating Ascorbic Acid Metabolism. Plant Physiology 184, 194-211.

Wu F., Qi J., Meng X. \& Jin W. (2020) miR319c acts as a positive regulator of tomato against Botrytis cinerea infection by targeting TCP29. Plant Science 300, 110610. 
1 Xie S., Jiang H., Xu Z., Xu Q. \& Cheng B. (2017) Small RNA profiling reveals important

2

3 roles for miRNAs in Arabidopsis response to Bacillus velezensis FZB42. Gene 629, 9-15.

4 Xu J., Hou Q.M., Khare T., Verma S.K. \& Kumar V. (2019) Exploring miRNAs for developing climate-resilient crops: A perspective review. Science of the Total Environment 653, 91-104.

Yang J.H., Han S.J., Yoon E.K. \& Lee W.S. (2006) 'Evidence of an auxin signal pathway, microRNA167-ARF8-GH3, and its response to exogenous auxin in cultured rice cells.' Nucleic Acids Research 34, 1892-1899.

Zhang B. (2015) MicroRNA: a new target for improving plant tolerance to abiotic stress. Journal of experimental botany 66, 1749-1761.

Zhang H. \& Sonnewald U. (2017) Differences and commonalities of plant responses to single and combined stresses. The Plant Journa/ 90, 839-855.

14 Zhao Y., Xie J., Wang S., Xu W., Chen S., Song X., ... Zhang D. (2021) Synonymous mutation of miR396a target sites in Growth Regulating Factor 15 (GRF15) enhances photosynthetic efficiency and heat tolerance in poplar. Journal of Experimental Botany.

Zhou M. \& Tang W. (2019) MicroRNA156 amplifies transcription factor-associated cold stress tolerance in plant cells. Molecular Genetics and Genomics 294, 379-393.

20 Zhou R., Yu X., Ottosen C.-O., Zhang T., Wu Z. \& Zhao T. (2020) Unique miRNAs and their targets in tomato leaf responding to combined drought and heat stress. BMC plant biology 20, 107.

23 Zhu Y.-X., Jia J.-H., Yang L., Xia Y.-C., Zhang H.-L., Jia J.-B., ... Liu L.-C. (2019) Identification of cucumber circular RNAs responsive to salt stress. BMC Plant Biology 19, 164. 


\section{FIGURE LEGENDS}

2 FIGURE 1 Analysis of the sRNA populations. (a) PCA based on sRNAs accumulation in 3 three biological replicates of melon plants exposed to the six stress combined 4 treatments and controls. The statistical significance $\left(p=5.886 \times 10^{-14}\right)$ was estimated by 5 Mann-Whitney-Wilcoxon test, considering the inter- and intra-group Euclidean 6 distances. (b) Diagram showing the relative accumulation (and distribution of the total 7 clean reads of melon sRNAs ranging between 20 - 25 nt obtained from the analyzed 8 sequenced libraries. The control and the different analyzed treatments are represented 9 with colors. The shown values represent the sum of all repetitions. Bars indicate the 10 standard error. (c) Graphic representation of the expression values (estimated by 11 edgeR) of sRNA sequences recovered from melon exposed to different stress 12 conditions. The dots indicate the expression value of each SRNA. Red and blue dots 13 indicate significant values for differential expression with $\left|\log _{2} F C\right| \geq 1.25$, respectively.

14 Grey dots indicate sRNAs with non-significant differential expression.

15

16 FIGURE 2 General description of stress-responsive miRNA families. (a) Boxplot 17 analysis showing the general expression value observed for each miRNA-family 18 member. To determine the general sense of the expression for each miRNA family we 19 employed the median value of expression (represented by internal box-line) estimated 20 by boxplot analysis of all family-related sequences. The differential expression values 21 represented in the figure correspond to the $\log _{2} F C$ obtained using edgeR. (b) Heatmap 22 of 22 miRNAs differentially expressed in melon in response to combined stress. The 23 differential expression values represented correspond to the median of the $\log _{2} F C$ 24 values obtained using edgeR for each miRNA family. (c) Scatter plot showing the 25 significant negative correlation (estimated by Pearson correlation coefficient) between 26 the expression levels of 16 selected stress-responsive miRNAs with differential 27 accumulation determined by sequencing and the accumulation of their targets in the 28 corresponding stress conditions, estimated by RT-qPCR.

30 FIGURE 3 Effects of the stresses combination onto the accumulation rate of stress 31 responsive miRNAs. (a) Graphic representation of the mean percentage for the six 32 analyzed treatments of miRNA related reads that exhibit additive (grey) or non-additive 33 (black) response to combined stress conditions in comparison to single stresses 34 considering unique reads (left columns), total reads (central columns) and miRNA 35 families (right). Bars represent the standard error between means. (b) Detail of the 36 global response rate in each stress condition considering the two (positive or negative) 37 type of possible non-additive response to combined stresses. (c) Volcano plot showing 38 significant positive (green dots) and negative (red dots) SCE values obtained for each 39 miRNA-related read, in response to each combined stress condition. miRNAs with non40 significant deviations from the additive null model are in grey. More detailed 41 information is provided in the Table S6B. 
2 FIGURE 4 Members of each miRNA family respond in a coordinated manner to 3 combined stresses. (a) Boxplot analysis showing the SCE values for family-miRNA 4 related members in each combined stress condition. To determine the general sense of 5 the effect induced by combined stresses for each miRNA family we employed the 6 median of the SCE values obtained for the totality of the family members (represented 7 by internal box-line). (b) Graphic representation of the global non-additive positive 8 (green) or negative (red) effects associated to combined stresses estimated for each 9 miRNA family in the six stress conditions analyzed here. The number of combined 10 stresses that induce positive and/or negative non-additive responses in each miRNA 11 family is detailed in the right columns. The proportion of miRNA families with non12 additive effects in response to each combined stresses is detailed below.

14 FIGURE 5 Biological functions of miRNAs with non-additive response to combined 15 stresses. (a) Dendrogram showing the clustering of miRNAs families with at least a 16 member with significant non-additive response to combined stresses in three main 17 groups according to their SCE values in the analyzed stress conditions. The global 18 statistical significance of the identified clusters $\left(p=8.88 \times 10^{-22}\right)$ was estimated by Mann19 Whitney-Wilcoxon test, considering the inter-and intra-group Euclidean distances. The 20 lower panel shows the response range determined for each miRNA family in response 21 to single stresses with both biotic and abiotic source (using a color scale). (b) 22 Description and detailed information of the targets for miRNAs with significant non23 additive response to combined stresses identified in melon plants. The GO terms were 24 estimated in base to information of homologous transcripts in $A$. thaliana.

26 FIGURE 6 Proposed model to explain predominant non-additive response in certain 27 miRNAs families. (a) Simplified graphic representation of the proposed miRNA28 mediated network of response to stress in melon (Sanz-Carbonell et al., 2019; Sanz29 Carbonell, Marques, Martinez \& Gomez, 2020). Blue nodes represent highly connected 30 miRNAs with a broad response range to biotic and/or abiotic stress conditions 31 (generalists). Orange nodes represent miRNAs reactive to specific stress conditions 32 (specialists). (b) When the network is exposed to double or triple stress conditions is 33 expected that the stresses combinations should not affect specialist miRNAs (poorly 34 connected between them) and consequently they exhibit additive SCE values 35 (comparable to the resultant of the sum of both individual responses). In contrast, 36 generalist miRNAs (highly interconnected) respond to stresses combination in a 37 differential (non-additive) manner, related to each stress combination. 


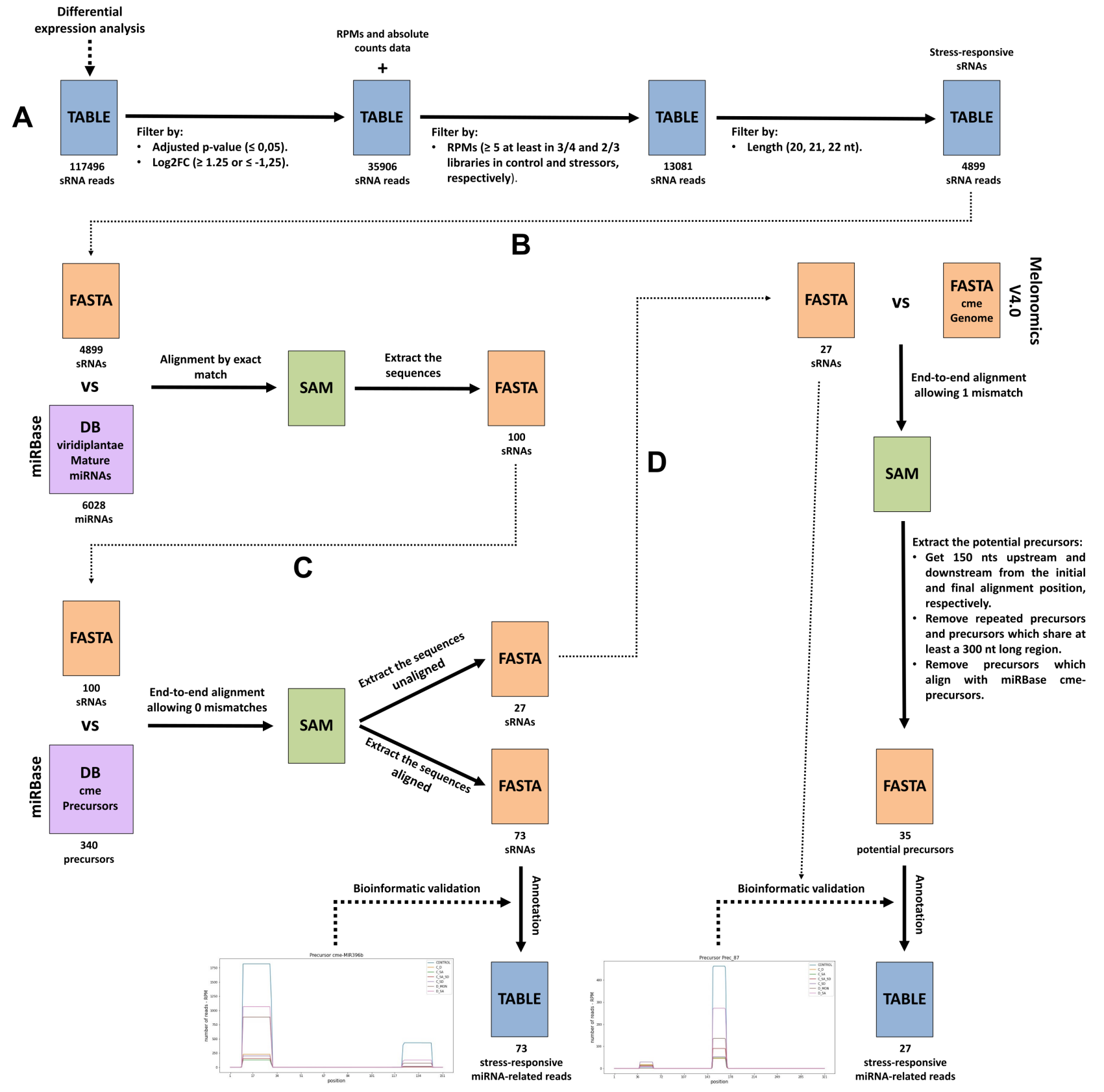

Figure S1. Pipeline for miRNA detection. A SRNA reads data coming from the differential expression analysis were filtered by adjusted $p$-value, log2FoldChange, RPMs and length to get the stress-responsive SRNA reads; B Stress-responsive sRNAs were aligned by exact match on viridiplantae mature miRNAs deposited in miRBase; C Stress-responsive sRNAs matched in the previous step were aligned on the cucumis melo precursors deposited in miRBase without allowing mismatches. The aligned sequences were bioinformatically validated and annotated as miRNA to be used in this work; D Stress-responsive sRNAs unaligned in the previous step were aligned on the cucumis melo genome regarding biological variability, that is, allowing 1 mismatch. Then, we looked for potential precursors which were used to bioinfomatically validate the sequences as miRNA. These sequences were annotated taking into account only the miRNA family of the viridiplantae mature miRNA on which aligned in the step B. 
Cold-Drought

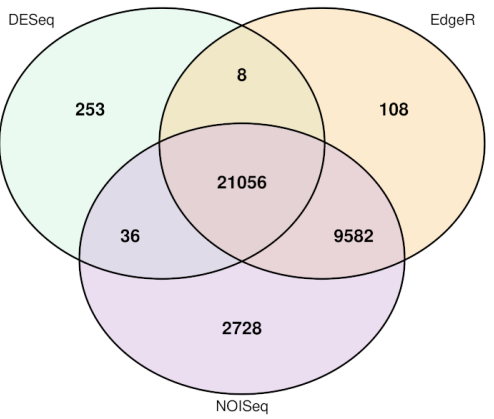

Drought-Monosporascus

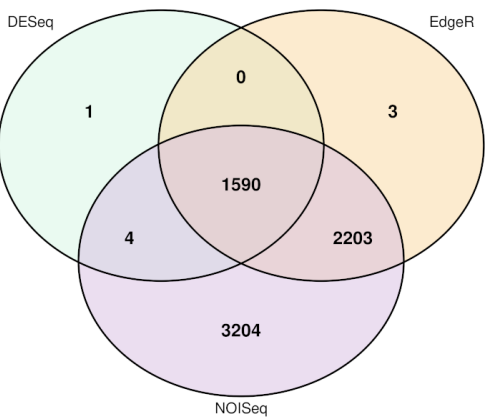

Cold-Salinity

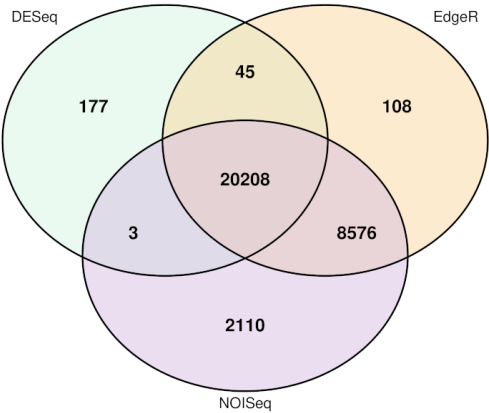

Drought-Salinity

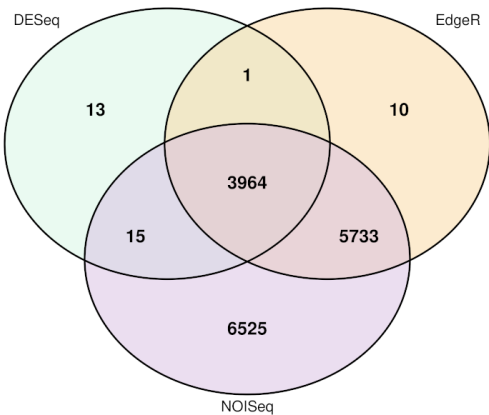

Cold-Short Day

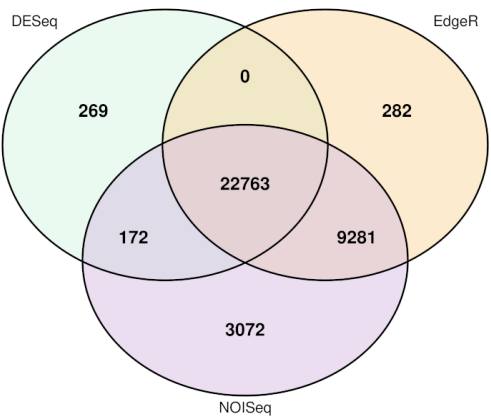

Cold-Salinity-Short Day

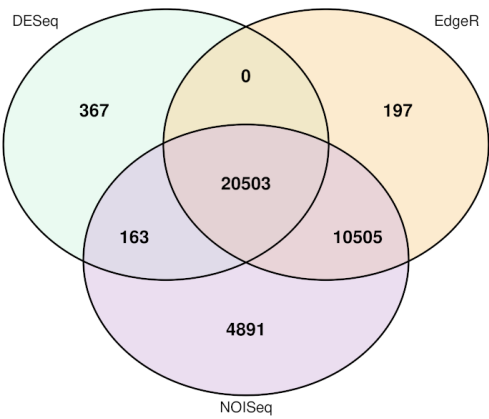

Figure S2: Analysis of stress-responsive miRNAs. Venn diagram comparing the number of the differential sRNAs estimated by DESeq2 (green), edgeR (orange) and NOISeq (magenta)- expressed in melon in response to combined stress conditions. Only the sRNAs predicted as differential by all three analysis methods were considered as true stress-responsive miRNAs. 
Table S1: Detail of the combined stress tratments used in this work.

\begin{tabular}{|c|c|c|c|}
\hline Stress Conditions & \multicolumn{2}{|c|}{ Treatments at 11 days post emergency } & sample collection \\
\hline Cold Drought & $\begin{array}{l}\text { Irrigated with } 50 \mathrm{ml} \text { Hoagland's solution } \\
20^{\circ} \mathrm{C} / 16 \mathrm{~h} \text {-light }--14^{\circ} \mathrm{C} / 8 \mathrm{~h} \text {-darkness }\end{array}$ & \multirow{7}{*}{$\begin{array}{l}\text { Except for drought combined } \\
\text { treatments plants were } \\
\text { irrigated alternatively (water } \\
\text { and Hoagland's solution) by } \\
\text { inundation ( } 1500 \mathrm{~mL} / 48 \mathrm{hs} \text { ) }\end{array}$} & \multirow{7}{*}{$\begin{array}{l}11 \text { days afetr stress } \\
\text { treatment }\end{array}$} \\
\hline Drought Salinity & $\begin{array}{l}\text { Irrigated with } 50 \mathrm{ml} \text { of } \mathrm{LiCl}(200 \mathrm{mM}) \\
28^{\circ} \mathrm{C} / 16 \text { h-light }---20^{\circ} \mathrm{C} / 8 \text { h-darkness } \\
\end{array}$ & & \\
\hline Cold Salinity & $\begin{array}{l}\text { Irrigated with } 50 \mathrm{ml} \text { of } \mathrm{LiCl}(200 \mathrm{mM}) \\
20^{\circ} \mathrm{C} / 16 \mathrm{~h} \text {-light }---14^{\circ} \mathrm{C} / 8 \text { h-darkness }\end{array}$ & & \\
\hline Cold Short-day & $\begin{array}{l}\text { Irrigated with } 50 \mathrm{ml} \text { Hoagland's solution } \\
20^{\circ} \mathrm{C} / 8 \text { h-light --- } 14^{\circ} \mathrm{C} / 16 \text { h-darkness } \\
\end{array}$ & & \\
\hline Drought Monosporascus & $\begin{array}{c}\text { Irrigated with } 50 \mathrm{ml} \text { Hoagland's solution plus } M \text {. } \\
\text { cannonballus mycelium (1000 UFC) } \\
28^{\circ} \mathrm{C} / 16 \text { h-light --- } 20^{\circ} \mathrm{C} / 8 \text { h-darkness } \\
\end{array}$ & & \\
\hline Cold Salinity Short-day & $\begin{array}{l}\text { Irrigated with } 50 \mathrm{ml} \text { of } \mathrm{LiCl}(200 \mathrm{mM}) \\
20^{\circ} \mathrm{C} / 8 \text { h-light }--4^{\circ} \mathrm{C} / 16 \text { h-darkness }\end{array}$ & & \\
\hline Control & $\begin{array}{l}\text { Irrigated with } 50 \mathrm{ml} \mathrm{Hoagland's} \mathrm{solution} \\
28^{\circ} \mathrm{C} / 16 \text { h-light }---20^{\circ} \mathrm{C} / 8 \text { h-darkness }\end{array}$ & & \\
\hline
\end{tabular}


Table 52:

Deatied intomation of control and strtess Sample SRNA length Library size Unique SRNAs Absolute counts RPMS Percentage \begin{tabular}{l|llllll}
\hline Control- 2 & 20 & 3254890 & 89809 & 207400 & 63719.51 & 6.37 \\
& 21 & 3254890 & 162375 & 462799 & 142185576 & 1422 \\
& 22 & 3 & & & &
\end{tabular} \begin{tabular}{rrrrrr}
22 & 3254890 & 158651 & 481894 & 148052.32 & 14.81 \\
\hline 23 & 3254890 & 231972 & 563459 & 17311.53 & 17.31 \\
\hline 24 & 3254890 & 8680012 & 133057 & 409555.16 & 40.96 \\
\hline
\end{tabular}

\begin{tabular}{ccccccc} 
& 24 & 3254890 & 868012 & 1333057 & 400555.16 & 40.96 \\
& 25 & 3254890 & 84481 & 206281 & 63375.72 & 6.34 \\
\hline Control-3 & 20 & 3638337 & 97378 & 199305 & 54779.15 & 5.48 \\
& 21 & 3638337 & 174735 & 464258 & 127601.70 & 12.76
\end{tabular}

\begin{tabular}{|c|c|c|c|c|}
\hline 20 & 3638337 & 97378 & 199305 & 54799.11 \\
\hline 21 & 3683337 & 174735 & 464258 & 127601.7 \\
\hline 22 & 3683337 & 169323 & 537064 & $14762=4$ \\
\hline & 3683337 & 254739 & 787518 & 216449.9 \\
\hline
\end{tabular}

\begin{tabular}{c|cccccc} 
& 24 & 3638337 & 938871 & 1443990 & 396881.87 & 39.69 \\
& 25 & 363837 & 84280 & 206202 & 56674.79 & 5.67 \\
\hline Control-4 & 20 & 4120101 & 110225 & 218788 & 53102.58 & 5.31
\end{tabular} \begin{tabular}{lllll}
520079 & 598124 & 145172.17 & 14.5 \\
\hline 40101 & 22079 &
\end{tabular}

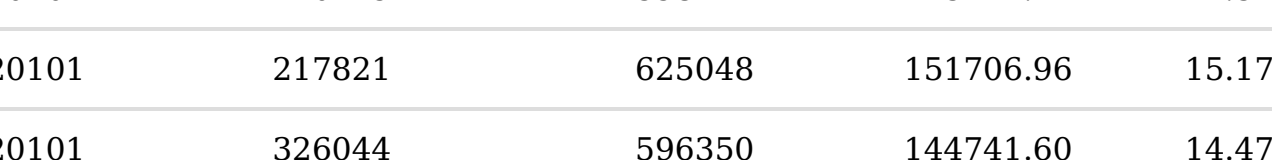

\begin{tabular}{c|cccccc} 
& 24 & 4120101 & 1180433 & 1847971 & 448525.66 & 44.85 \\
\hline & 25 & 4120101 & 109378 & 233820 & 5671.04 & 5.68 \\
\hline Control.5 & 20 & 309997 & 74339 & 168072 & 54216.83 & 5.42 \\
\hline & 21 & 3099997 & 147609 & 441991 & 142577.88 & 1426
\end{tabular}

\begin{tabular}{|c|c|c|c|c|c|c|}
\hline & 22 & 3099997 & 140915 & 478706 & 154421.44 & 15.44 \\
\hline & 23 & 3099997 & 201649 & 685367 & 221086.34 & 22.11 \\
\hline & 24 & 3099997 & 746109 & 1147989 & 370319.39 & 37.03 \\
\hline & 25 & 309997 & 68633 & 177872 & 57378.12 & 5.74 \\
\hline$\overline{\overline{c-D}-1}$ & 20 & 3535271 & 933672 & 256127 & 72449.04 & 7.24 \\
\hline
\end{tabular}

\begin{tabular}{|c|c|c|c|c|c|c|}
\hline \multirow{6}{*}{ C-D.-1 } & 20 & 3552771 & 93672 & 256127 & 72449.04 & 7.24 \\
\hline & 21 & 3535271 & 163192 & 510797 & 144485.95 & 1445 \\
\hline & 22 & 3535271 & 163994 & 420383 & 118991.111 & 11.89 \\
\hline & 23 & 3535271 & 220670 & 420602 & 118997.06 & 11.90 \\
\hline & 24 & 3555271 & 790983 & 1488811 & 421132.92 & 42.11 \\
\hline & 25 & 3555271 & 91011 & 438543 & 124447.92 & 12.40 \\
\hline 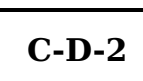 & ${ }^{20}$ & 4476774 & 103973 & 231692 & 51754.23 & $\begin{array}{l}5.18 \\
\end{array}$ \\
\hline
\end{tabular}

\begin{tabular}{|c|c|c|c|c|c|c|}
\hline \multirow[t]{6}{*}{ C-D-2 } & 20 & 4476774 & 103973 & 231692 & 51754.23 & 5.18 \\
\hline & 21 & 4476774 & 199393 & 571488 & 127656.21 & 12.77 \\
\hline & 22 & 4476774 & 202429 & 535414 & 119598.17 & 11.96 \\
\hline & 23 & 4476774 & 273691 & 470427 & 105081.69 & 10.51 \\
\hline & 24 & 4476774 & 1058297 & 2178447 & 486610.89 & 48.66 \\
\hline & 25 & 4476774 & 103661 & 489306 & 109298.79 & 10.93 \\
\hline \multirow[t]{3}{*}{ C-D-D.3 } & 20 & 31677043 & 83415 & 188210 & 59427.67 & 5.94 \\
\hline & 21 & 3167043 & 153710 & 447131 & 141182.48 & 14.12 \\
\hline & 22 & 3167043 & 151266 & 367009 & 115883.81 & 11.59 \\
\hline
\end{tabular}

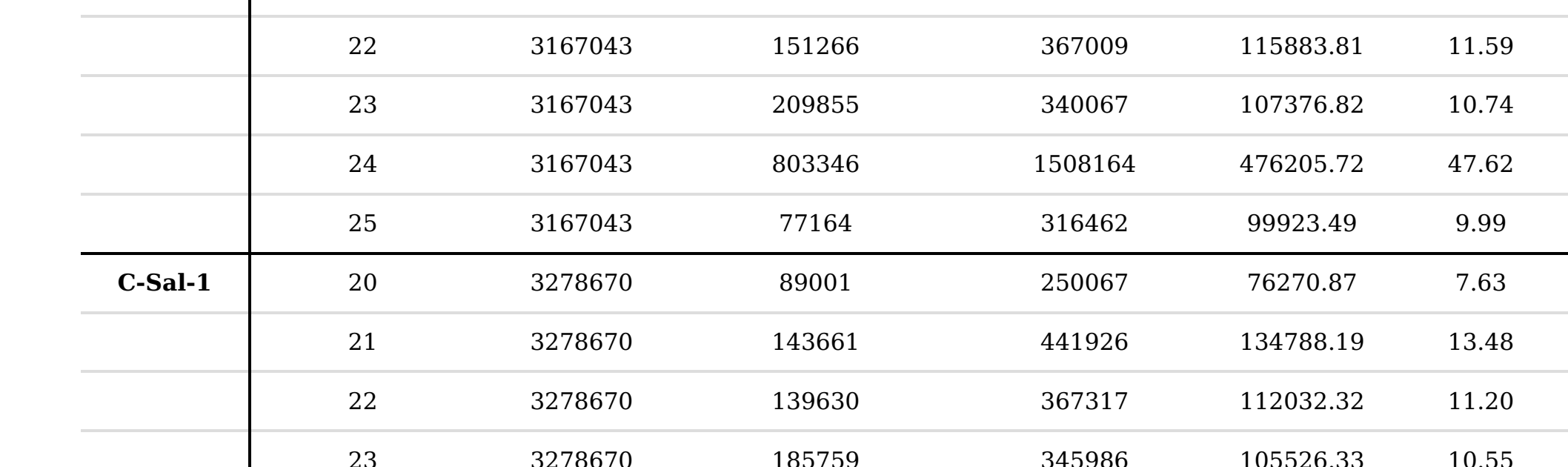

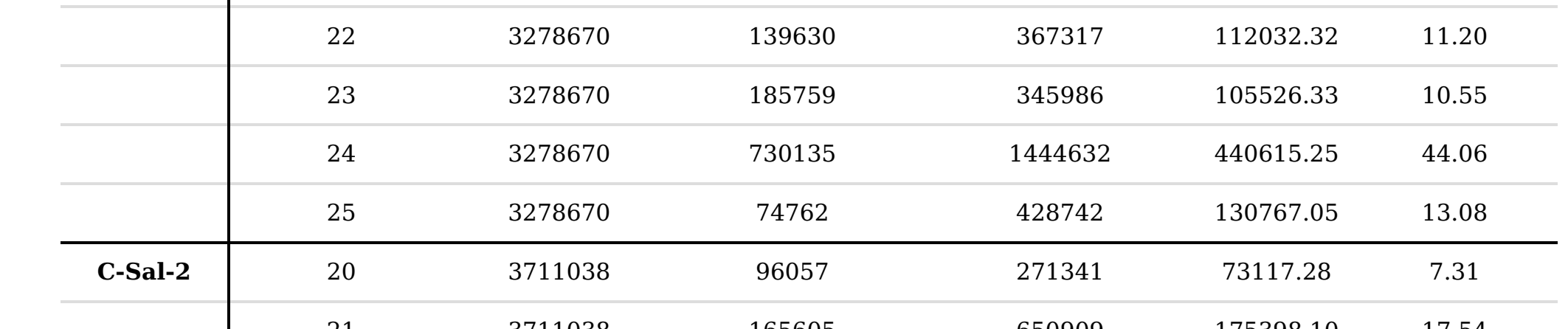

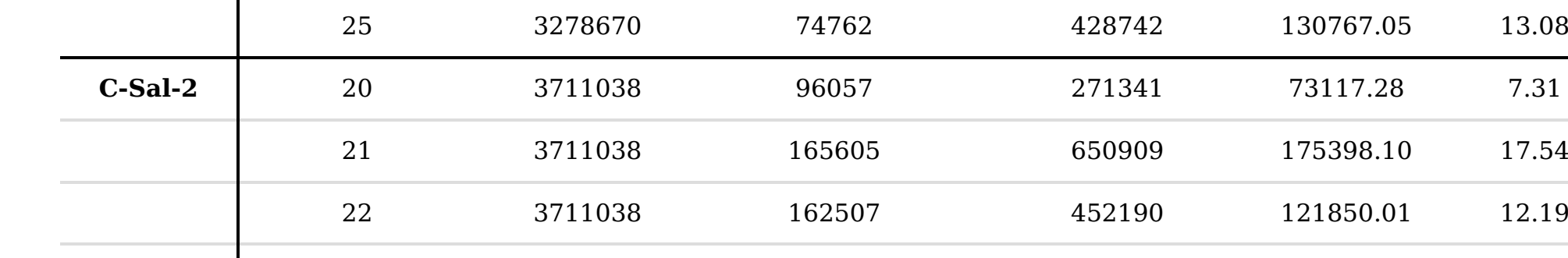

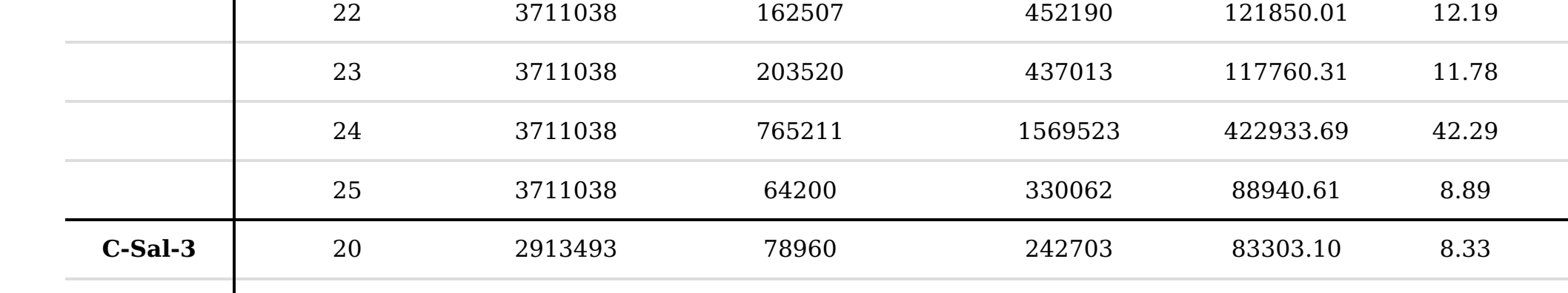

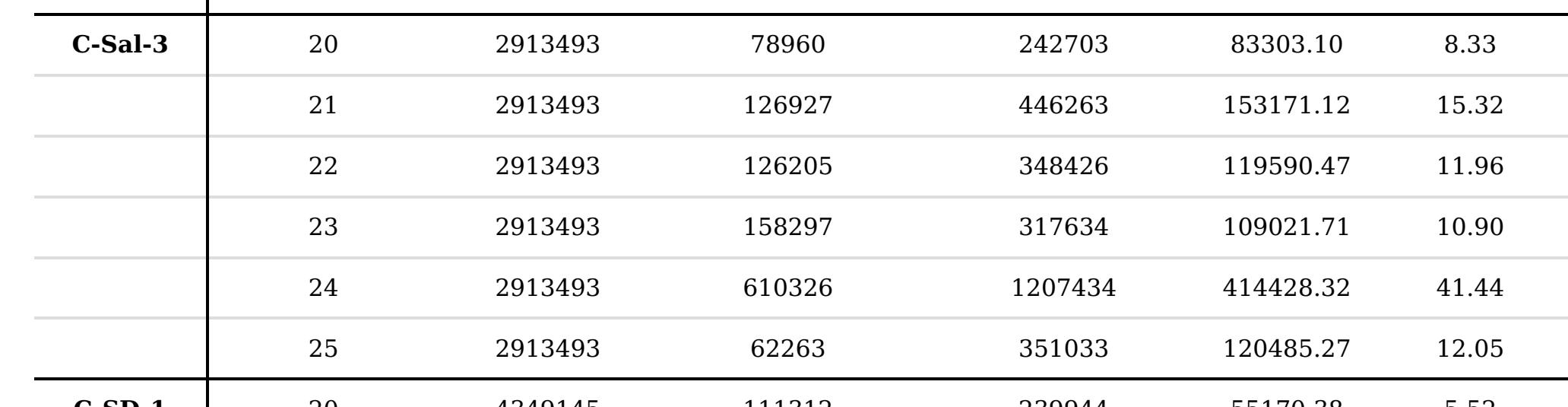

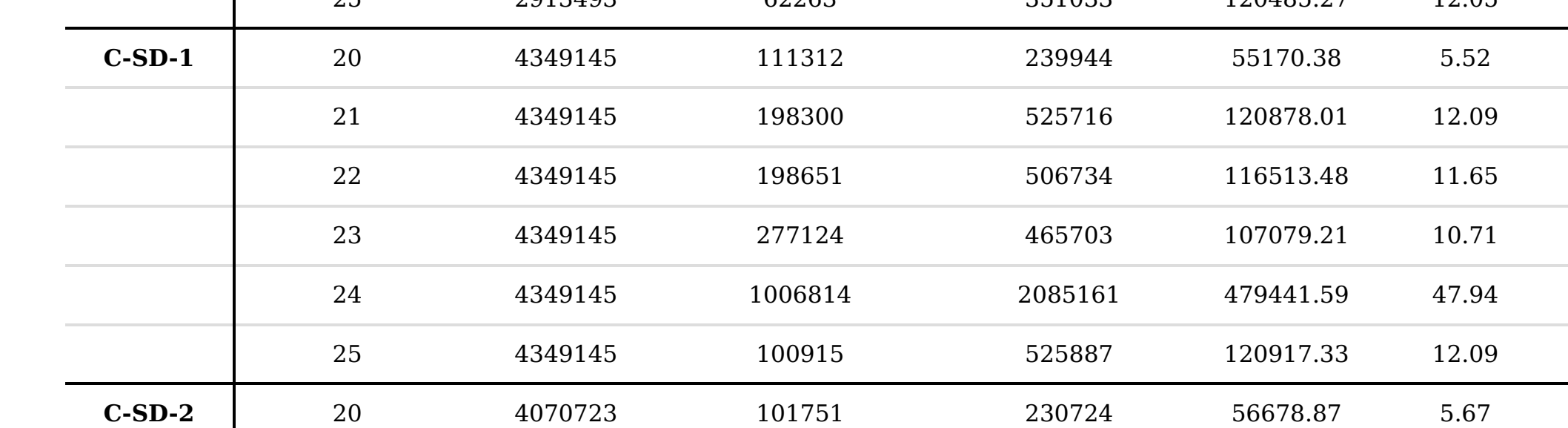

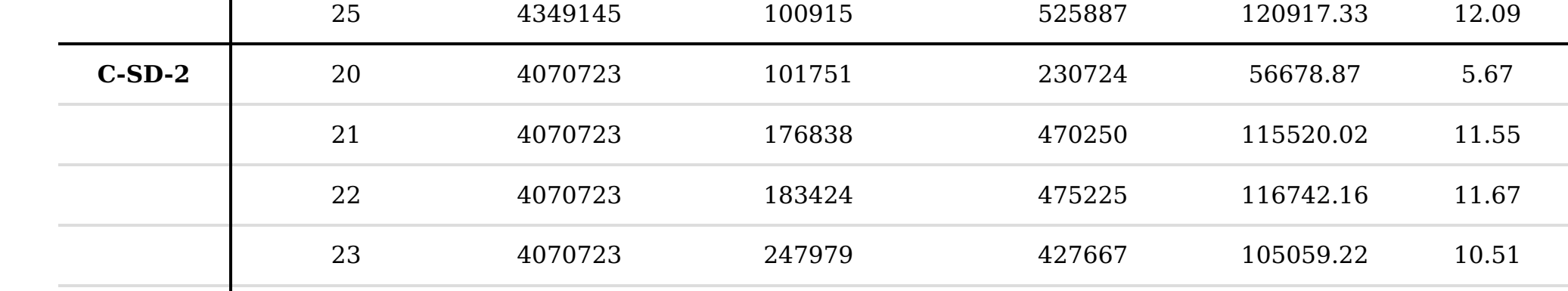

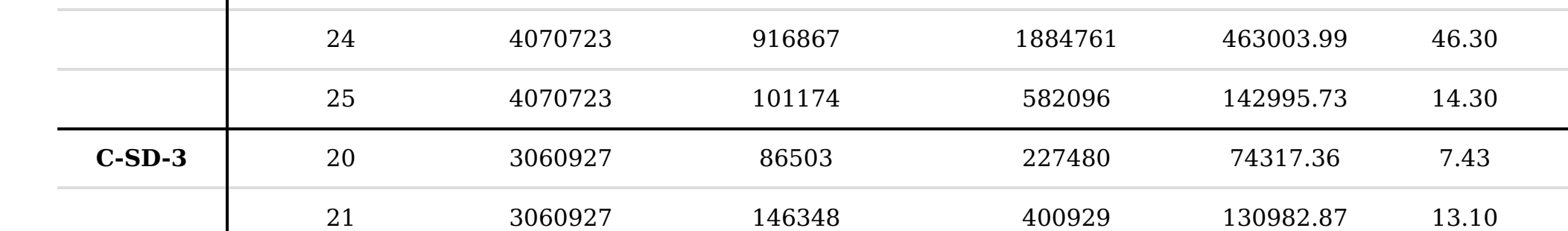

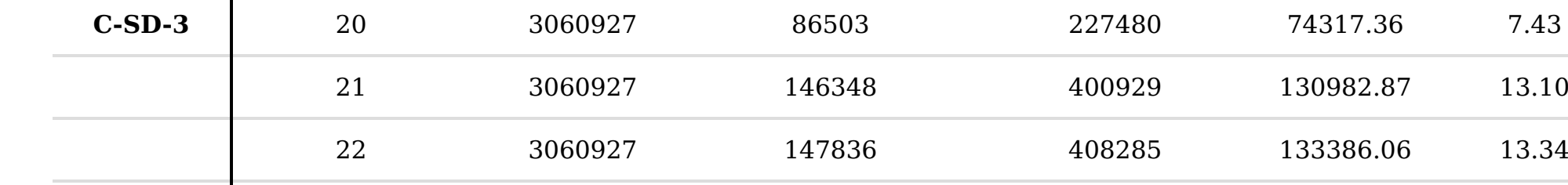

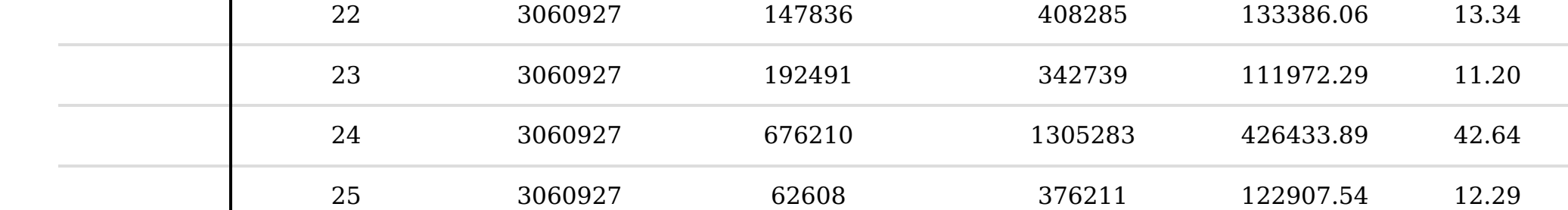

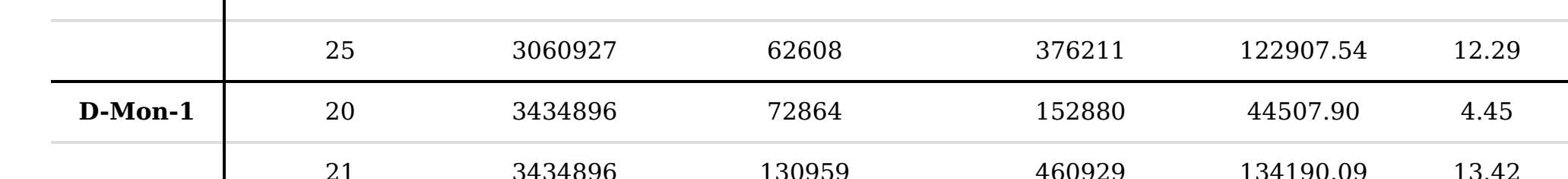

\begin{tabular}{llllll}
21 & 3434896 & 130959 & 460929 & 1341190.09 & 13.42 \\
\hline 22 & 3434896 & 144773 & 521031 & 151687.56 & 15.17 \\
\hline & & & & &
\end{tabular}

\begin{tabular}{llllll}
34348966 & 234176 & 687786 & 200234.88 & 15.17 \\
\hline & 20.02 \\
\hline
\end{tabular}

\begin{tabular}{lllll}
3434896 & 883783 & 1436127 & 41809.12 & 41.81 \\
\hline
\end{tabular}

\begin{tabular}{l|llllll} 
& 25 & 3434896 & 73586 & 176143 & 51280.45 & 5.13 \\
\hline D.Mon-2 & 20 & 2621444 & 62174 & 128036 & 48841.78 & 4.88 \\
& 21 & 2621444 & 111173 & 355345 & 135553.15 & 13.56
\end{tabular}

$\begin{array}{llllll}21 & 2621444 & 111173 & 355345 & 135553.15 & 13.55 \\ 22 & 2621444 & 115707 & 372087 & 141939.71 & 14.1 \\ 2 & & & & & \end{array}$

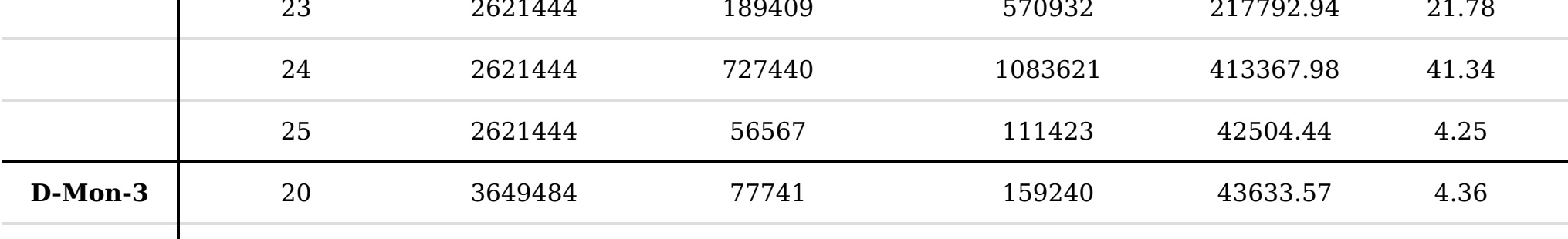

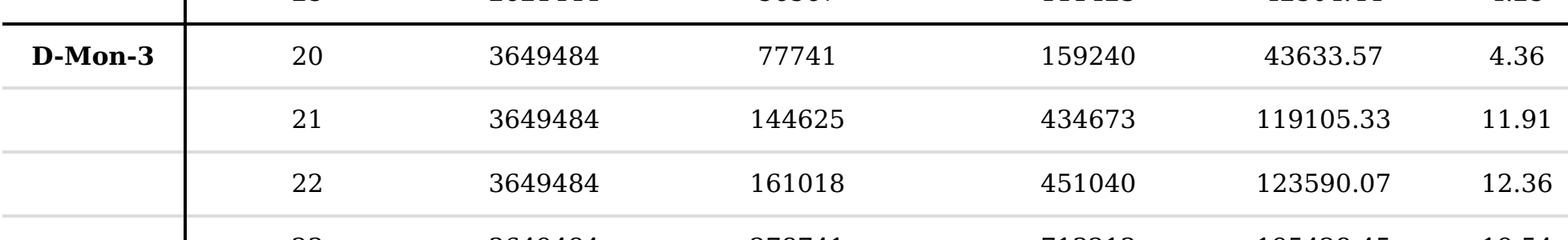

$\begin{array}{lllll}3669484 & 1073308 & 1716823 & 47042899 & 49.54 \\ & & 4704\end{array}$

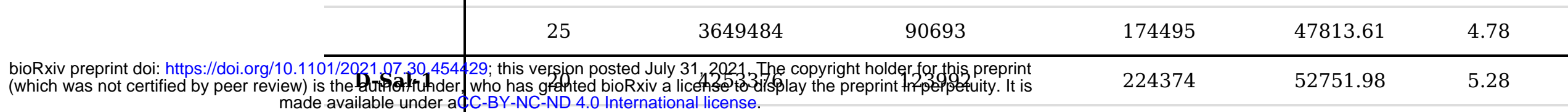

\begin{tabular}{|c|c|c|c|c|c|c|}
\hline & 21 & 42535377 & 214434 & 551760 & 129972.84 & 12.97 \\
\hline & 22 & 4253377 & 213687 & 568311 & 133614.10 & 13.36 \\
\hline & 23 & 4253377 & 297266 & 572260 & 134542.54 & 13.45 \\
\hline & 24 & 42533776 & 1298718 & 2106407 & 495231.79 & 49.52 \\
\hline & 25 & 4253376 & 124654 & 230264 & 54136,76 & 5.41 \\
\hline \multirow[t]{6}{*}{ D-Sal-2 } & 20 & 3565226 & 1066153 & 244490 & 68193.75 & 6.82 \\
\hline & 21 & 3565226 & 171457 & 477700 & 131567.72 & 13.16 \\
\hline & 22 & 3565226 & 1773928 & 476265 & 132841.00 & 13.28 \\
\hline & 23 & 3565226 & 243561 & 422040 & 117716.43 & 11.77 \\
\hline & 24 & 3565226 & 932902 & 1753949 & 489215.74 & 48.92 \\
\hline & 25 & 3565226 & 114080 & 216782 & 60465.37 & 6.05 \\
\hline \multirow[t]{6}{*}{ D.Sal-3 } & 20 & 344560 & 1044040 & 233128 & 67880.05 & 6.77 \\
\hline & 21 & 344560 & 171156 & 489739 & 1422177.52 & 14.22 \\
\hline & 22 & 344560 & 1677156 & 430935 & 1255105.96 & 12.51 \\
\hline & 23 & 344560 & 228695 & 393395 & 114207,62 & 11.42 \\
\hline & 24 & 344560 & 902364 & 1696530 & 492524.44 & 49.25 \\
\hline & 25 & 344560 & 105199 & 200833 & 5830440 & 5.83 \\
\hline \multirow[t]{6}{*}{ c-Sal-sD-1 } & 20 & 4138669 & 101646 & 2169941 & 52418.06 & 5.24 \\
\hline & 21 & 4138669 & 192739 & 555918 & 134866.14 & 13.48 \\
\hline & 22 & 4138669 & 185482 & 448279 & 100374.77 & 10.83 \\
\hline & ${ }^{23}$ & 4138669 & 264682 & 435175 & 105148.54 & 10.51 \\
\hline & 24 & 4138669 & 1110437 & 2255499 & 54981.73 & 54.50 \\
\hline & 25 & 4138669 & 84308 & 224857 & 54330.75 & 5.43 \\
\hline \multirow[t]{6}{*}{ c-Sal-sD-2 } & 20 & 4483072 & 86074 & 220464 & 49176.99 & 4.92 \\
\hline & 21 & 4483072 & 178532 & 576425 & 1228578.1.12 & 12.86 \\
\hline & 22 & 4483072 & 1798999 & 462350 & 1033132.41 & 10.31 \\
\hline & ${ }_{23}$ & 4483072 & 255651 & 447117 & 99734.51 & 9.97 \\
\hline & 24 & 4483072 & 1154005 & 2306011 & 514381.88 & 51.44 \\
\hline & 25 & 4483072 & 97719 & 470705 & 104996.08 & 10.50 \\
\hline \multirow{6}{*}{ C-Sal-sD-3 } & 20 & 4333858 & 94571 & 225872 & 52118.00 & 5.21 \\
\hline & 21 & 4333858 & 185569 & 592924 & 136612.05 & 13.68 \\
\hline & 22 & 4333858 & 180488 & 445661 & 102832.40 & 10.28 \\
\hline & ${ }^{23}$ & 4333858 & 245468 & 43030304 & 99288.90 & 9.93 \\
\hline & 24 & 4333858 & 1107161 & 2254538 & 520215.01 & 52.02 \\
\hline & 25 & 4333858 & 97867 & 384559 & 88733.64 & \\
\hline
\end{tabular}




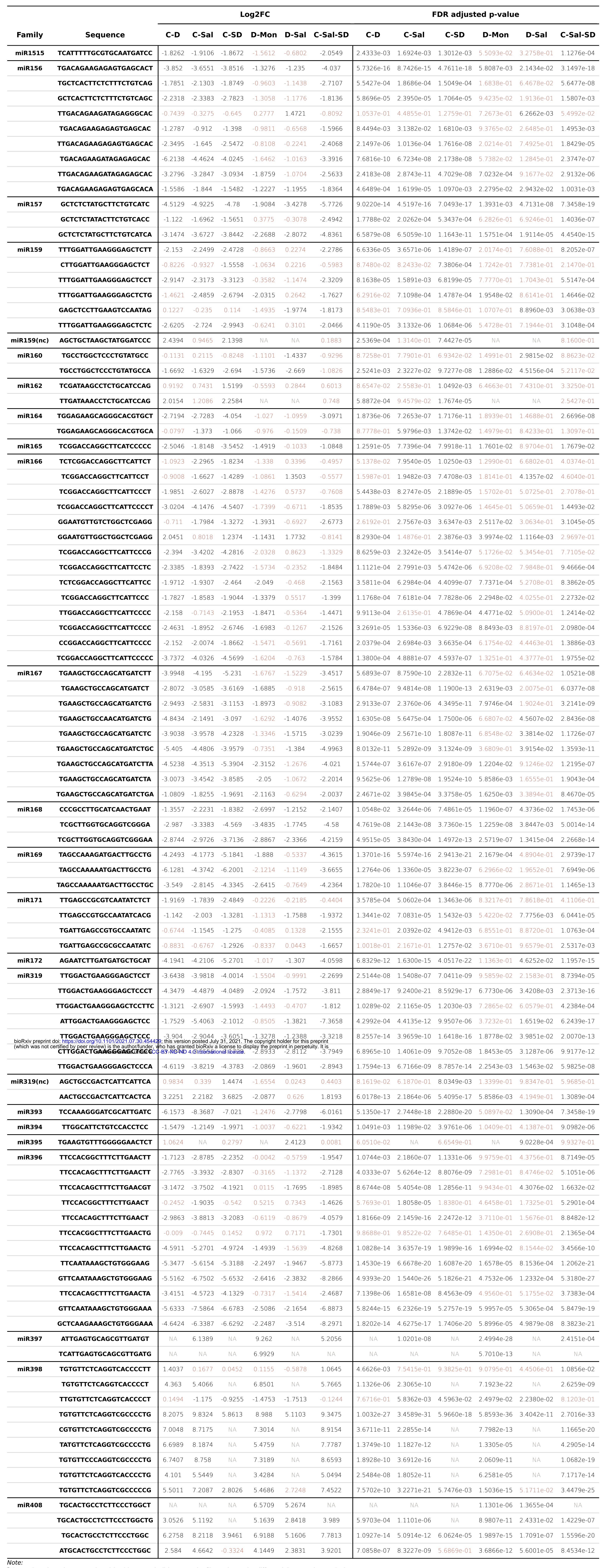


Table S3a: Statically analysis of sRNAs-reads profiles in control and stresses exposed plants. The differences between treatment and readslength were analyzed by the Scheirer-Ray-Hare non-parametric test (upper). Once established that only length category shown significant alterations we used Dunn's Multiple Comparison Test to analyze the difference between $24 \mathrm{nt}$ length reads and the rest of the read-size categories (lower).

\begin{tabular}{ccccc} 
Categories & Df & Sum Sq & H & $p$.value \\
\hline Stress & 6 & 3808 & 2.603 & 0.85676 \\
Length & 5 & 144878 & 99.028 & $<1.0 \mathrm{E}-5$ \\
Stress:Length & 30 & 35813 & 24.479 & 0.74993
\end{tabular}

Size

\begin{tabular}{cccc} 
Comparison & Z & $p . u n a d j$ & $p . a d j$ \\
\hline $20-24$ & $-8,994$ & $2,38 \mathrm{E}-19$ & $3,57 \mathrm{E}-18$ \\
$21-24$ & $-3,323$ & $8,92 \mathrm{E}-04$ & $1,34 \mathrm{E}-02$ \\
$22-24$ & $-4,316$ & $1,59 \mathrm{E}-05$ & $2,38 \mathrm{E}-04$ \\
$23-24$ & $-4,643$ & $3,43 \mathrm{E}-06$ & $5,15 \mathrm{E}-05$ \\
$25-24$ & 7,339 & $2,15 \mathrm{E}-13$ & $3,23 \mathrm{E}-12$
\end{tabular}


Table S4:

Presence and absence of stress-responsive miRNAs to a combined stress conditions in Cucumis melo.

1: stress-responsive, 0 : non stress-responsive.

\begin{tabular}{|c|c|c|c|c|c|c|c|}
\hline Family & C-D & C-Sal & C-SD & D-Mon & D-Sal & C-Sal-SD & Total \\
\hline $\operatorname{miR156}$ & 1 & 1 & 1 & 1 & 1 & 1 & 6 \\
\hline $\operatorname{miR} 157$ & 1 & 1 & 1 & 1 & 1 & 1 & 6 \\
\hline $\operatorname{miR} 159$ & 1 & 1 & 1 & 1 & 1 & 1 & 6 \\
\hline $\operatorname{miR166}$ & 1 & 1 & 1 & 1 & 1 & 1 & 6 \\
\hline $\operatorname{miR167}$ & 1 & 1 & 1 & 1 & 1 & 1 & 6 \\
\hline $\operatorname{miR} 168$ & 1 & 1 & 1 & 1 & 1 & 1 & 6 \\
\hline $\operatorname{miR319}$ & 1 & 1 & 1 & 1 & 1 & 1 & 6 \\
\hline $\operatorname{miR396}$ & 1 & 1 & 1 & 1 & 1 & 1 & 6 \\
\hline $\operatorname{miR398}$ & 1 & 1 & 1 & 1 & 1 & 1 & 6 \\
\hline $\operatorname{miR408}$ & 1 & 1 & 1 & 1 & 1 & 1 & 6 \\
\hline $\operatorname{miR} 160$ & 1 & 1 & 1 & 1 & 1 & 0 & 5 \\
\hline $\operatorname{miR} 169$ & 1 & 1 & 1 & 1 & 0 & 1 & 5 \\
\hline $\operatorname{miR171}$ & 1 & 1 & 1 & 0 & 1 & 1 & 5 \\
\hline $\operatorname{miR393}$ & 1 & 1 & 1 & 0 & 1 & 1 & 5 \\
\hline $\operatorname{miR1515}$ & 1 & 1 & 1 & 0 & 0 & 1 & 4 \\
\hline $\operatorname{miR} 164$ & 1 & 1 & 1 & 0 & 0 & 1 & 4 \\
\hline $\operatorname{miR} 172$ & 1 & 1 & 1 & 0 & 0 & 1 & 4 \\
\hline $\operatorname{miR165}$ & 1 & 1 & 1 & 0 & 0 & 0 & 3 \\
\hline $\operatorname{miR394}$ & 1 & 0 & 1 & 0 & 0 & 1 & 3 \\
\hline $\operatorname{miR397}$ & 0 & 1 & 0 & 1 & 0 & 1 & 3 \\
\hline $\operatorname{miR} 162$ & 1 & 0 & 1 & 0 & 0 & 0 & 2 \\
\hline miR395 & 0 & 0 & 0 & 0 & 1 & 0 & 1 \\
\hline Total & 20 & 19 & 20 & 13 & 14 & 18 & 104 \\
\hline
\end{tabular}




\begin{tabular}{|c|c|c|c|}
\hline \multirow{2}{*}{$\begin{array}{l}\text { target-miRNA } \\
\text { module }\end{array}$} & \multirow{2}{*}{ stress } & \multicolumn{2}{|c|}{ miRNA Target } \\
\hline & & UE & UE \\
\hline \multirow{8}{*}{ mik156-Sp19 } & 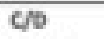 & 2,35 & 3,41 \\
\hline & crse & -4.64 & 2,12 \\
\hline & $C / S D$ & -2.55 & 3,20 \\
\hline & D/sa & 1.67 & $1 . n$ \\
\hline & D/MON & -1.88 & 1,67 \\
\hline & crseasso & 2,41 & 2,62 \\
\hline & $\mathrm{cro}$ & -2.15 & 2,97 \\
\hline & crsa & -2.25 & 4,01 \\
\hline
\end{tabular}

bioRxiv preprint doi: https://doi.org/10.1101/2021.07.30.454429; this version posted July 31,2021 . The copyright holder for this preprint (which was not certified by peer review) is the author/funder, who has granted bioRxiv a license to display the preprint in perpetuity. It is made available under aCC-BY-NC-ND 4 .0 International license.

\begin{tabular}{|c|c|c|c|}
\hline \multirow{5}{*}{ miR160-AEF17 } & Clo & -1.67 & 1.69 \\
\hline & cisa & $-1,63$ & 2,16 \\
\hline & C/so & .2 .69 & 1,82 \\
\hline & Drsa & -2.67 & 0,38 \\
\hline & D/MON & -2.57 & 2.34 \\
\hline \multirow{4}{*}{$\min 164 \mathrm{NAC}$} & $C D$ & $2, \pi$ & 0,05 \\
\hline & cise & 2,73 & 0,95 \\
\hline & c/so & $-4,05$ & $-0, \pi 9$ \\
\hline & Crsarso & -3.10 & 1.57 \\
\hline \multirow{6}{*}{ miR166-ATHB14 } & CD & 2.46 & 5.26 \\
\hline & crsa & $-1,90$ & 6,75 \\
\hline & c/so & $-2,67$ & 6,35 \\
\hline & D/se & in & 2,70 \\
\hline & D/MON & -1.70 & 6.51 \\
\hline & crsenso & 2.15 & 5.24 \\
\hline \multirow{6}{*}{ miR167-Aar6 } & Clo & $-2,96$ & 1,59 \\
\hline & crsa & .258 & 1,57 \\
\hline & c/sD & -1.12 & 1.54 \\
\hline & D/sa & $-1,57$ & $-0,50$ \\
\hline & D/MON & $-1,90$ & 1,51 \\
\hline & c/serso & $-3,11$ & 1,50 \\
\hline \multirow{5}{*}{ miR169-NFY } & $\mathrm{ClO}$ & 4.25 & 1.44 \\
\hline & c/sis & 4.18 & 2,07 \\
\hline & Csso & $-5,18$ & 1,27 \\
\hline & D/MeN & $-1,89$ & 1,87 \\
\hline & C/se/so & $-4,36$ & 1,64 \\
\hline \multirow{5}{*}{ mial71-scl6 } & co & -1.92 & 3,67 \\
\hline & casa & 2,00 & 4,52 \\
\hline & CSO & 2,48 & 3,81 \\
\hline & D/sa & $-4,76$ & 1.66 \\
\hline & crsarso & -2.84 & 3.49 \\
\hline \multirow{4}{*}{ mik172-AP2 } & 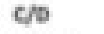 & 4.19 & 2,64 \\
\hline & cisa & $4 n$ & 4,06 \\
\hline & $c / s 0$ & $-5,27$ & 3,50 \\
\hline & c/sa/so & $-4,06$ & 3,95 \\
\hline \multirow{6}{*}{$\min 319-\mathrm{TCP} 2$} & Clo & 4.35 & 3.06 \\
\hline & cssa & 4,49 & 3,03 \\
\hline & coso & 4,05 & 2,96 \\
\hline & D/sa & $-1,76$ & 0,60 \\
\hline & D/MON & -2.00 & 1,40 \\
\hline & crsasso & -181 & 2.56 \\
\hline \multirow{5}{*}{ mik393-ASB2 } & 00 & 4,16 & 0,91 \\
\hline & crsa & $-4,37$ & 1,54 \\
\hline & $c / s 0$ & $-7,02$ & 1.04 \\
\hline & D/M & -2.78 & 0,35 \\
\hline & crsarso & 6.00 & 1,15 \\
\hline \multirow{6}{*}{ miR396-GRF9 } & $c 0$ & 2,78 & $2, \pi$ \\
\hline & crsa & $-3,39$ & 3,19 \\
\hline & C/SD & -2.83 & 2.36 \\
\hline & ora & $-2,38$ & 0,32 \\
\hline & D/MON & $-1,49$ & 1,30 \\
\hline & crsa/so & 2,71 & 2,99 \\
\hline \multirow{3}{*}{ mik397-4sp } & crsa & 6.14 & 2,06 \\
\hline & D/MON & 9.26 & 0.53 \\
\hline & crsarso & 5.21 & 2.19 \\
\hline & 60 & 8.21 & $-0,32$ \\
\hline & c/sa & 9.83 & $-0,51$ \\
\hline miras cup & C/so & 5.65 & 0.51 \\
\hline miR398CUP & orsa & 5.11 & 1,33 \\
\hline & D/MoN & 8,99 & $-0,03$ \\
\hline & crsarso & 9,35 & $-0,82$ \\
\hline & $C / 0$ & .21 & $-0,39$ \\
\hline & Cras & 2.83 & $-1,15$ \\
\hline maygesob & CAso & 5.26 & 0,19 \\
\hline & b/M & 5.11 & 0,20 \\
\hline & B/MCN & 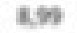 & -0.83 \\
\hline & cravso & 2.13 & $-0,61$ \\
\hline & $C 10$ & 2.5 & $-2,16$ \\
\hline & CMA & 4.66 & $-2,60$ \\
\hline milaces at? & CNo & $1 \times$ & $-2,17$ \\
\hline mencesere & D/A & 2.48 & 1.24 \\
\hline & D/MON & 4.14 & $-1,19$ \\
\hline & cravse & 192 & 1.75 \\
\hline
\end{tabular}


Table 56B:

Log10(SCE + 1) values of stress-responsive miRNAs in Cucumis melo.

\begin{tabular}{|c|c|c|c|c|c|c|c|}
\hline Family & SRNA & C-D & C-Sal & C-SD & D-Mon & D-Sal & C-Sal-SD \\
\hline $\operatorname{miR} 1515$ & TCATTTTTGCGTGCAATGATCC & 0.205 & -0.5775 & 0.4546 & -0.7541 & -0.6381 & -0.79 \\
\hline \multirow[t]{7}{*}{ miR156 } & GCTCACTTCTCTTTCTGTCAGC & 0.0829 & -0.3162 & -0.2046 & -0.5491 & -0.622 & -0.6858 \\
\hline & TGACAGAAGAGAGTGAGCACA & 0.9932 & -0.9311 & 0.6012 & -0.4497 & -0.9554 & -1.3785 \\
\hline & TGACAGAAGAGAGTGAGCACT & 0.856 & -0.9707 & 0.7265 & -0.7899 & -1.1356 & -1.4622 \\
\hline & TGСTCAСTTCTСTTTСTGTCAG & 2.2955 & -1.4007 & 1.5016 & 2.0962 & 1.7082 & -2.5608 \\
\hline & TTGACAGAAGAGAGTGAGCAC & 2.1891 & -2.6423 & 1.1456 & -2.1953 & -2.5281 & -3.0071 \\
\hline & TTGACAGAAGATA & 1.4373 & -1.7235 & 0.2556 & -1.3264 & -1.6538 & -2.0545 \\
\hline & TTGACAGAAGATAGAGGGCAC & -0.8374 & -1.4539 & -0.7914 & -0.9082 & -0.9924 & -1.6467 \\
\hline \multirow[t]{3}{*}{$\operatorname{miR} 157$} & GCTCTCTATACTTCTGTCACC & 0.9692 & -1.1521 & -1.3609 & 1.2435 & -0.5613 & -1.7005 \\
\hline & GCTCTCTATGCTTCTGTCATC & 2.8414 & 2.6475 & 2.4262 & 2.8407 & 2.6665 & -1.966 \\
\hline & GCTCTCTATGCTTCTGTCATCA & 1.6437 & 1.3138 & -0.8241 & 1.5289 & 1.3492 & -1.5833 \\
\hline \multirow[t]{6}{*}{$\operatorname{miR} 159$} & AGCTGCTAAGCTATGGATCCC & 0.547 & -0.4997 & 0.3673 & 0.1305 & -0.0939 & -0.652 \\
\hline & GAGCTCCTTGAAGTCCAATAG & 0.6604 & -0.5027 & -0.9771 & 0.4558 & -0.2673 & -1.3769 \\
\hline & TTTGGATTGAAGGGAGCTCCT & -0.0334 & -0.5602 & 0.2852 & -0.1555 & -0.6839 & -0.66 \\
\hline & TTTGGATTGAAGGGAGCTCTC & -0.9226 & -1.3389 & 0.6968 & -1.2269 & -1.1464 & -1.4662 \\
\hline & TTTGGATTGAAGGGAGCTCTG & 0.0085 & -1.4011 & -0.6886 & -1.3266 & 1.911 & -1.5097 \\
\hline & TTTGGATTGAAGGGAGCTCTT & -1.4546 & -1.9506 & 1.3783 & -1.9287 & -1.5142 & -2.1776 \\
\hline $\operatorname{miR} 160$ & TGCCTGGCTCCCTGTATGCCA & 1.4254 & 0.5763 & -1.1352 & 0.7522 & -1.4112 & -1.5688 \\
\hline \multirow[t]{2}{*}{$\operatorname{miR} 162$} & TCGATAAGCCTCTGCATCCAG & -0.7613 & -0.7768 & 0.2137 & -0.7026 & -0.039 & -0.9202 \\
\hline & TTGATAAACCTCTGCATCCAG & 0.2356 & -0.5735 & 0.4711 & -0.2702 & -0.0318 & -0.7952 \\
\hline $\operatorname{miR} 164$ & TGGAGAAGCAGGGCACGTGCA & 0.6826 & -0.8174 & -0.4465 & -0.6793 & -0.677 & -1.0665 \\
\hline & TGGAGAAGCAGGGCACGTGCT & -0.9737 & -1.1474 & 0.4214 & -1.2283 & -1.462 & -1.3768 \\
\hline miR165 & TCGGACCAGGCTTCATCCCCC & -0.296 & -1.1319 & 0.31 & -1.1089 & -1.1324 & -1.2174 \\
\hline miR166 & CCGGACCAGGCTTCATTCCCC & 0.1345 & -0.8522 & 0.5157 & -0.8992 & -0.8886 & -1.0415 \\
\hline & GGAATGTTGGCTGGCTCGAGG & 1.8061 & -1.9876 & -0.9263 & -1.6505 & -1.3736 & -2.2517 \\
\hline & GGAATGTTGTCTGGCTCGAGG & 1.7935 & -2.0966 & -1.056 & -1.8381 & -2.0101 & -2.3977 \\
\hline & TCGGACCAGGCTTCATTCCCC & 1.7759 & -3.4511 & 2.6882 & -3.4721 & -3.4195 & -3.6796 \\
\hline & TCGGACCAGGCTTCATTCCCCC & 0.6952 & -1.4369 & 1.4086 & -1.2601 & -1.5238 & -1.3379 \\
\hline & TCGGACCAGGCTTCATTCCCCT & 1.8536 & -2.4579 & 2.4163 & -2.0164 & -2.4177 & -2.3491 \\
\hline & TCGGACCAGGCTTCATTCCCG & -1.0515 & $-1,2472$ & 0.9904 & -1.639 & 0.8597 & -0.9963 \\
\hline & TCGGACCAGGCTTCATTCCCT & -2.6359 & -3.1931 & 2.3874 & -3.1117 & -3.1234 & -3.1315 \\
\hline & TCGGACCAGGCTTCATTCCTC & -0.5458 & -0.8649 & -0.2474 & -0.978 & -0.9974 & -1.0853 \\
\hline & TCTCGGACCAGGCTTCATTCC & 1.8999 & -2.2848 & -0.5772 & -2.2991 & -2.1836 & -2.6313 \\
\hline & TCTCGGACCAGGCTTCATTCT & -1.3555 & -2.0793 & 1.1213 & -1.9835 & -1.9444 & -2.0008 \\
\hline & TTGGACCAGGCTTCATTCCCC & 0.3075 & 0.3428 & 0.42 & -0.6439 & -0.4393 & -0.5037 \\
\hline miR167 & TGAAGCTGCCAACATGATCTG & 0.1039 & -0.1901 & 0.1852 & -0.4533 & -0.3785 & -0.598 \\
\hline & TGAAGCTGCCAGCATGATCTA & 1.8078 & -2.4246 & 2.1449 & -1.8587 & -2.4274 & -2.4603 \\
\hline & TGAAGCTGCCAGCATGATCTC & 0.4004 & -1.2635 & 1.1037 & -1.0892 & -1.4278 & -1.3517 \\
\hline & TGAAGCTGCCAGCATGATCTG & 2.9393 & -3.3545 & 3.0106 & -3.2857 & -3.3652 & -3.6118 \\
\hline & TGAAGCTGCCAGCATGATCTGA & 0.0871 & -0.7048 & 0.3552 & -0.9288 & -0.7209 & -0.8748 \\
\hline & TGAAGCTGCCAGCATGATCTGC & -0.0653 & -0.6987 & 0.3939 & -0.6173 & -0.8166 & -0.829 \\
\hline & TGAAGCTGCCAGCATGATCTT & 1.0557 & -1.7963 & 1.6886 & -1.4518 & -1.968 & -1.9198 \\
\hline & TGAAGCTGCCAGCATGATCTTA & -0.2589 & -0.7729 & 0.4087 & -0.7222 & -0.8964 & -0.8683 \\
\hline $\operatorname{miR168}$ & CCCGCCTTGCATCAACTGAAT & 2.1358 & -1.8696 & 1.7239 & 1.1692 & 1.1938 & -2.2298 \\
\hline & TCGCTTGGTGCAGGTCGGGAA & 2.2302 & -0.904 & 1.9011 & 1.8964 & -0.9668 & -2.1238 \\
\hline $\operatorname{miR} 169$ & TAGCCAAAAATGACTTGCCTG & 0.45 & -0.8258 & 0.45 & 0.0288 & -0.7344 & -0.8105 \\
\hline & TAGCCAAAAATGACTTGCCTGC & 0.715 & -1.3313 & 0.6278 & -0.9566 & -1.3059 & -1.4487 \\
\hline & 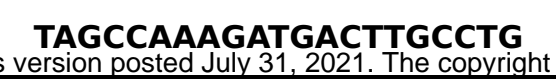 & & & 0.5322 & -1.0592 & -0.8824 & -1.2212 \\
\hline 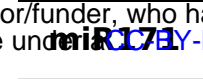 & 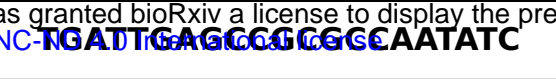 & & wity. II & -0.5161 & -1.2123 & -1.239 & -1.4447 \\
\hline & TGATTGAGCCGTGCCAATATC & 0.4273 & -0.5566 & -0.6952 & -0.7 & -0.534 & -1.1871 \\
\hline & TTGAGCCGCGTCAATATCTCT & -0.4265 & -0.8882 & 0.2802 & -0.535 & -0.9628 & -0.865 \\
\hline & TTGAGCCGTGCCAATATCACG & 0.3336 & -0.995 & 0.2087 & -1.2716 & -1.3224 & -1.556 \\
\hline $\operatorname{miR} 172$ & AGAATCTTGATGATGCTGCAT & 0.931 & -0.4473 & -1.311 & -0.2753 & -0.4512 & -1.641 \\
\hline miR319 & AАCTGCCGACTCATTCACTCA & 0.991 & -1.1321 & 1.3643 & -0.5028 & 0.3612 & -1.3234 \\
\hline & AGCTGCCGACTCATTCATTCA & 0.4746 & -0.4442 & 0.7579 & -0.2195 & 0.6453 & -0.5187 \\
\hline & CTTGGACTGAAGGGAGCTCCC & 0.8094 & 0.1356 & -0.2261 & 0.2183 & -0.1413 & -0.8651 \\
\hline & TTGGACTGAAGGGAGCTCCCA & 0.9825 & 0.4082 & 0.8014 & 0.7648 & 0.578 & -0.5567 \\
\hline & TTGGACTGAAGGGAGCTCCCT & 2.0399 & 1.2766 & 1.5234 & 1.0002 & 1.3769 & -2.119 \\
\hline & TTGGACTGAAGGGAGCTCCTTC & 0.3422 & -0.073 & -0.0672 & -0.1475 & 0.5444 & -0.4171 \\
\hline miR393 & TCCAAAGGGATCGCATTGATC & 0.3709 & -0.2638 & -0.6522 & -0.3379 & -0.9032 & -1.1661 \\
\hline miR395 & TGAAGTGTTTGGGGGAACTCT & 0.4485 & -0.6927 & -0.1711 & 0.2736 & 0.6788 & -0.7418 \\
\hline miR396 & GCTCAAGAAAGCTGTGGGAAA & 0.7803 & -0.3349 & -0.6452 & 0.4826 & -0.5847 & -1.1031 \\
\hline & GTTCAATAAAGCTGTGGGAAA & 1.1695 & -0.1022 & 1.0304 & 1.1126 & 0.5173 & -0.9273 \\
\hline & GTTCAATAAAGCTGTGGGAAG & 2.511 & 1.7002 & 2.1458 & 2.1873 & 2.0012 & -2.3082 \\
\hline & TTCCACAGCTTTCTTGAACGT & 0.7188 & 0.496 & 0.6126 & 0.6516 & -0.323 & -0.3943 \\
\hline & TTCCACAGCTTTCTTGAACTA & 2.1384 & 1.4162 & 2.1265 & 2.1764 & 1.5871 & -1.6342 \\
\hline & TTCCACAGCTTTCTTGAACTG & 2.8058 & -1.3804 & 2.6742 & 1.5958 & 1.9194 & -2.8109 \\
\hline & TTCCACAGCTTTCTTGAACTT & 3.5025 & 3.1677 & 3.1526 & 3.4072 & 3.3923 & -3.6048 \\
\hline & TTCCACGGCTTTCTTGAACTG & -1.9932 & -1.992 & -1.707 & -1.5926 & 1.8041 & -2.5032 \\
\hline & TTCCACGGCTTTCTTGAACTT & 1.0057 & 0.6049 & 0.6131 & 1.1164 & 1.2166 & -1.0678 \\
\hline miR397 & TCATTGAGTGCAGCGTTGATG & -1.1728 & 0.3905 & 0 & -1.0048 & -1.1728 & 0 \\
\hline miR398 & CGTGTTCTCAGGTCGCCCCTG & -0.9672 & 1.2052 & -0.6492 & -0.6467 & -1.0143 & 1.2303 \\
\hline & TATGTTCTCAGGTCGCCCCTG & -0.1284 & 1.0873 & -0.5448 & -0.2717 & -0.5399 & 0.8997 \\
\hline & TGTGTTCCCAGGTCGCCCCTG & -0.9798 & 1.2463 & -0.6256 & -0.6607 & -0.9816 & 1.1638 \\
\hline & TGTGTTCTCAGGTCACCCCTG & -0.4704 & 1.1877 & -0.7261 & -0.4834 & -0.7611 & 1.0627 \\
\hline & TGTGTTCTCAGGTCACCCCTT & 0.9448 & -0.9462 & -0.8554 & 0.16 & -0.5903 & -0.7453 \\
\hline & TGTGTTCTCAGGTCGCCCCCG & -1.5124 & 1.3931 & -1.2382 & -1.2769 & -1.4087 & 1.5699 \\
\hline & & -3.4215 & 3.6181 & -2.976 & -3.1014 & -3.4686 & 3.4992 \\
\hline & & 1.0342 & -0.7886 & -0.2349 & $-4 \mathrm{e}-04$ & -0.5018 & 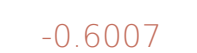 \\
\hline miR408 & ATGCACTGCCTCTTCCCTGGC & -3.2175 & 2.8169 & -1.7641 & -3.1514 & -3.21 & 2.6228 \\
\hline & & 1.204 & 0.456 & & -1.0953 & -1.1574 & 0.364 \\
\hline & & & & & & 1.441 & \\
\hline
\end{tabular}


Table S7: Detail of the percentage of additive and non-additive values SCE values obtained for differentially expressed miRNAs in each analyzed stress combination.

\section{Stress combination effects}

unique reads total reads miRNA families

Additive No Additive Additive No Additive Additive No Additive

\begin{tabular}{ccccccc} 
C-D & 75,9 & 24,1 & 37,27 & 62,73 & 59,09 & 40,91 \\
C-Sal & 62,65 & 37,35 & 45,73 & 54,28 & 36,36 & 63,64 \\
C-SD & 85,54 & 14,46 & 92,24 & 7,77 & 59,09 & 40,91 \\
D-Mon & 63,86 & 36,14 & 63,01 & 36,99 & 36,36 & 63,64 \\
D-Sal & 65,06 & 34,94 & 54,26 & 45,74 & 40,91 & 59,09 \\
C-Sal-SD & 38,55 & 61,45 & 7,95 & 92,05 & 22,73 & 77,27 \\
\cline { 2 - 7 } & & & & & \\
Mean in & 65,26 & 34,74 & 50,08 & 49,93 & 42,42 & 57,58 \\
all stress & & & & & &
\end{tabular}




\section{Table S7:}

Presence and absence of Stress Combination Effect (SCE) for stressresponsive miRNAs in Cucumis melo.

1: non-additive SCE, 0 : additive SCE.

\begin{tabular}{|c|c|c|c|c|c|c|c|}
\hline Family & C-D & C-Sal & C-SD & D-Mon & D-Sal & C-Sal-SD & Total \\
\hline $\operatorname{miR156}$ & 1 & 1 & 1 & 1 & 1 & 1 & 6 \\
\hline $\operatorname{miR} 157$ & 1 & 1 & 1 & 1 & 1 & 1 & 6 \\
\hline $\operatorname{miR319}$ & 1 & 1 & 1 & 1 & 1 & 1 & 6 \\
\hline $\operatorname{miR396}$ & 1 & 1 & 1 & 1 & 1 & 1 & 6 \\
\hline $\operatorname{miR398}$ & 1 & 1 & 1 & 1 & 1 & 1 & 6 \\
\hline $\operatorname{miR} 159$ & 0 & 1 & 1 & 1 & 1 & 1 & 5 \\
\hline $\operatorname{miR166}$ & 1 & 1 & 0 & 1 & 1 & 1 & 5 \\
\hline $\operatorname{miR167}$ & 0 & 1 & 1 & 1 & 1 & 1 & 5 \\
\hline $\operatorname{miR408}$ & 1 & 0 & 1 & 1 & 1 & 1 & 5 \\
\hline $\operatorname{miR} 171$ & 0 & 1 & 0 & 1 & 1 & 1 & 4 \\
\hline $\operatorname{miR} 164$ & 0 & 1 & 0 & 1 & 1 & 1 & 4 \\
\hline $\operatorname{miR165}$ & 0 & 1 & 0 & 1 & 1 & 1 & 4 \\
\hline $\operatorname{miR} 168$ & 1 & 1 & 0 & 0 & 0 & 1 & 3 \\
\hline $\operatorname{miR169}$ & 0 & 1 & 1 & 0 & 1 & 0 & 3 \\
\hline $\operatorname{miR} 172$ & 1 & 0 & 0 & 0 & 0 & 1 & 2 \\
\hline $\operatorname{miR395}$ & 0 & 1 & 0 & 0 & 0 & 1 & 2 \\
\hline $\operatorname{miR1515}$ & 0 & 0 & 0 & 0 & 1 & 1 & 2 \\
\hline $\operatorname{miR393}$ & 0 & 0 & 0 & 1 & 0 & 0 & 1 \\
\hline $\operatorname{miR} 162$ & 0 & 0 & 0 & 0 & 0 & 1 & 1 \\
\hline $\operatorname{miR397}$ & 0 & 0 & 0 & 0 & 0 & 0 & 0 \\
\hline $\operatorname{miR} 160$ & 0 & 0 & 0 & 0 & 0 & 0 & 0 \\
\hline $\operatorname{miR394}$ & 0 & 0 & 0 & 0 & 0 & 0 & 0 \\
\hline Total & 9 & 14 & 9 & 13 & 14 & 17 & 76 \\
\hline
\end{tabular}

